ISSN 1855-3966 (printed edn.), ISSN 1855-3974 (electronic edn.)

ARS MATHEMATICA CONTEMPORANEA 22 (2022) \#P2.10

https://doi.org/10.26493/1855-3974.2433.ba6

(Also available at http://amc-journal.eu)

\title{
Characterization of a family of rotationally symmetric spherical quadrangulations
}

\author{
Lowell Abrams \\ The George Washington University, Washington DC 20052, USA \\ Daniel Slilaty * \\ Wright State University, Dayton $\mathrm{OH} 45435$, USA
}

Received 11 September 2020, accepted 29 August 2021, published online 27 May 2022

\begin{abstract}
A spherical quadrangulation is an embedding of a graph $G$ in the sphere in which each facial boundary walk has length four. Vertices that are not of degree four in $G$ are called curvature vertices. In this paper we classify all spherical quadrangulations with $n$-fold rotational symmetry $(n \geq 3)$ that have minimum degree 3 and the least possible number of curvature vertices, and describe all such spherical quadrangulations in terms of nets of quadrilaterals. The description reveals that such rotationally symmetric quadrangulations necessarily also have a pole-exchanging symmetry.
\end{abstract}

Keywords: Quadrangulation, spherical quadrangulation, rotational symmetry.

Math. Subj. Class. (2020): 05C10

\section{Introduction}

If $S$ is a closed surface, a graph $G$ embedded in $S$ in which all facial boundary walks have length four is called a quadrangulation of $S$. When $S$ is the sphere, the graph $G$ is necessarily bipartite. Considering quadrilateral faces to be geometrically flat squares, vertices of degree 4 extend this flatness to neighboring faces. Thus, when "most" of the vertices of a spherical quadrangulation are of degree four, large areas will appear as a portion of the geometrically-flat, infinite $\{4,4\}$-planar lattice. The curvature is therefore localized at vertices of degree other than 4 .

In spherical triangulations where each face is considered to be a flat equilateral triangle, vertices of degree 6 play a similar role in extending flatness, and curvature is thus localized

\footnotetext{
*Corresponding author.

E-mail addresses: labrams@gwu.edu (Lowell Abrams), daniel.slilaty@wright.edu (Daniel Slilaty)
} 


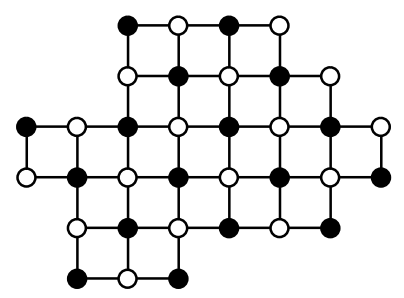

Figure 1: A flat region with all internal vertices of degree 4.

at vertices of degree other than 6 . Such spherical triangulations link to several well-studied structures; for example, those for which all of the curvature is localized at 12 vertices of degree 5 were popularized by the geodesic domes of Buckminster Fuller. Moreover, these triangulations were later noted to be the topological dual graphs of what are now called fullerene graphs. ([4] is a good starting source for fullerenes and other chemical graphs.)

As such, in a quadrangulation of $S$, a vertex of degree other than 4 is called a curvature vertex. In this paper we investigate spherical quadrangulations with $n$-fold rotational symmetry ( $n \geq 3$ ) that have minimum degree 3 and the least possible number of curvature vertices, which is 8 when $n=4$ and $2 n+2$ otherwise. (See Proposition 2.1 and Corollary 2.2.) The fact that curvature is localized at a relatively small number of vertices suggests that $G$ may have a description in terms of a geometric net of polygons. For example, in Figure 2 we have a net of six congruent quadrilaterals which closes up to yield a spherical quadrangulation with 3 -fold rotational symmetry about poles $p$ and $q$. Note that there are $2 n+2=8$ curvature vertices of degree 3 each.

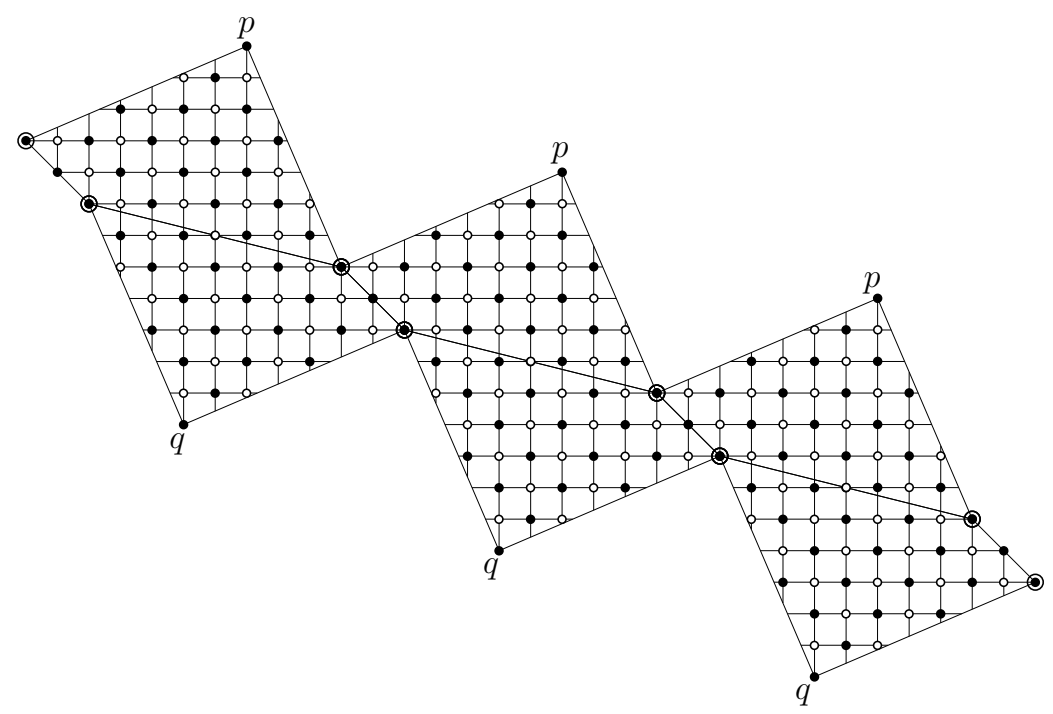

Figure 2: A quadrilateral net which describes a spherical quadrangulation with 3-fold rotational symmetry.

Our main result (Theorem 1.1) is that every such spherical quadrangulation has a similar 
description as a very simple net of $2 n$ congruent quadrilaterals. The Three- and FourParameter Constructions mentioned in Theorem 1.1 are stated in full detail in Section 3. We also identify which of these nets produce quadrangulations that are overlay graphs of self-dual planar embeddings.

Theorem 1.1. Let $G$ be a spherical quadrangulation with minimum degree 3 , having $n$-fold rotational symmetry $(n \geq 3)$, and having the least possible number of curvature vertices. If

(1) all white vertices have degree 4 and

(2) the poles of the rotational symmetry are at two black vertices,

then $G$ can be obtained from either the Three- or Four-Parameter Construction of Section 3.

Remark 1.2. The reader may note that not all spherical quadrangulations with $n$-fold rotational symmetry and the least possible number of curvature vertices satisfy Conditions (1) and (2) of Theorem 1.1. Nevertheless, any such spherical quadrangulation can still be obtained using Theorem 1.1 by making use of Proposition 1.3. In short, even if $G$ does not satisfy both (1) and (2), it is necessarily the case that $G$ overlayed with its topological dual graph will be a spherical quadrangulation which does satisfy both (1) and (2). This will be described in more detail in Section 1.1 in the paragraph after Proposition 1.3.

The reader may note that the quadrangulation of Figure 2, and indeed all of the quadrangulations constructed in Section 3, not only possesses $n$-fold rotational symmetry at poles $p$ and $q$ but also has a 2-fold symmetry which exchanges $p$ and $q$. This is interesting in that this 2 -fold symmetry is not a priori implied by our hypotheses. Hence our spherical quadrangulations possess, at the very least, an order- $2 n$ symmetry group. Any such quadrangulations possessing additional symmetries will, of course, also be included in our constructions.

\subsection{Overlay graphs and other background on quadrangulations}

An important fact about quadrangulations is that a spherical quadrangulation is always bipartite while quadrangulations of other surfaces need not be. Given any graph $H$ that is cellularly embedded in a closed surface $S$, two bipartite quadrangulations that are naturally associated with the embedding of $H$ and its topological dual graph $H^{*}$ are the overlay graph and the radial graph. For the sphere, in fact, any quadrangulation $G$ is a radial graph for some embedding $H$ and its dual $H^{*}$. Applications of radial graphs, overlay graphs, and the closely associated medial graph can be found in $[1,2,3,5,7,8,9]$.

Consider a graph $H$ cellularly embedded in a closed surface $S$ and also consider its topological dual graph $H^{*}$. Say that all of $H$ (both vertices and edges) is colored "red" and all of $H^{*}$ is colored "blue". Embed $H$ and $H^{*}$ simultaneously in $S$ and at each edge/dualedge crossing point create a new vertex of degree four (which now has alternating red and blue edges in rotation around it) and say that this new vertex is "white". The graph obtained is called the overlay graph $\mathcal{O}\left(H, H^{*}\right)$. Note that $H$ is self dual if and only if $\mathcal{O}\left(H, H^{*}\right)$ has a cellular automorphism which leaves white invariant and switches red and blue colors.

The overlay graph $\mathcal{O}\left(H, H^{*}\right)$ was used by Servatius and Servatius [8] to classify selfdual embeddings in the sphere along with the pairing of their groups of color-preserving cellular automorphisms of $\mathcal{O}\left(H, H^{*}\right)$ as index-2 subgroups of the groups of the whitepreserving cellular automorphisms. Graver and Hartung [5] do the same in the special case 
of self-dual embeddings of graphs having four trivalent vertices and the remaining vertices all of degree four. Their results, however, are much more explicit than those in [8].

The overlay graph $\mathcal{O}\left(H, H^{*}\right)$ is a bipartite quadrangulation of a closed surface $S$ with partite sets Red $\cup$ Blue and White. The radial graph of $H$ and $H^{*}$ (denote it by $\mathcal{R}\left(H, H^{*}\right)$ ) can be constructed from $\mathcal{O}\left(H, H^{*}\right)$ by placing a diagonal edge in each face of $\mathcal{O}\left(H, H^{*}\right)$ which connects the red and blue vertices on that face and then erasing all of the edges and white vertices of $\mathcal{O}\left(H, H^{*}\right)$. Thus $\mathcal{R}\left(H, H^{*}\right)$ is also a bipartite quadrangulation of $S$. Conversely, if $G$ is a bipartite quadrangulation of $S$ with bipartition Red $\cup$ Blue, then $G=\mathcal{R}\left(H, H^{*}\right)$ for some $H$ and $H^{*}$ as follows. In each face of $G$, place a red edge connecting the two red vertices and a blue edge connecting the two blue vertices. The resulting red and blue graphs are $H$ and $H^{*}$.

Unlike the radial graph, even if $G$ is a bipartite quadrangulation of $S$ in which all white vertices have degree four it is not necessarily true that $G$ is of the form $\mathcal{O}\left(H, H^{*}\right)$ for some $H$. An additional condition that does ensure that $G$ has the form $\mathcal{O}\left(H, H^{*}\right)$ is given in Proposition 1.3. In Proposition 1.3, $\mathcal{D}(G)$ is the graph obtained from quadrangulation $G$ by placing a diagonal edge connecting the black corners of each face and then deleting the white vertices of $G$. As mentioned in the previous paragraph, $\mathcal{D}(G)$ is the radial graph for some $K$ and $K^{*}$ in $S$ when $\mathcal{D}(G)$ is bipartite.

Proposition 1.3. If $G$ is a quadrangulation of closed surface $S$, then $G=\mathcal{O}\left(H, H^{*}\right)$ for some $H$ if and only if every white vertex of $G$ has degree 4 and both $G$ and $\mathcal{D}(G)$ are bipartite. In the particular case that $S$ is the sphere, $G=\mathcal{O}\left(H, H^{*}\right)$ for some $H$ if and only if every white vertex of $G$ has degree 4.

Proof. First, let $S$ be any closed surface. The one direction of the equivalence statement is trivial. For the other direction, the fact that $\mathcal{D}(G)$ is bipartite allows us to properly 2-color (red and blue) the vertices of $\mathcal{D}(G)$, which shows $G$ is of the form $\mathcal{O}\left(H, H^{*}\right)$, as required.

The statement for the sphere follows from the first statement and the fact that any spherical quadrangulation is automatically bipartite.

Now say $H$ is a spherical quadrangulation with $n$-fold rotational symmetry and the minimum number of curvature vertices, but does not satisfy the other two conditions in Theorem 1.1. In this case $\mathcal{O}\left(H, H^{*}\right)$ inherits the $n$-fold rotational symmetry of $H$ and does satisfy the two additional conditions.

Quadrangulations have also been studied for other surfaces. Thomassen [10] and also Márquez, de Mier, Noy, Revuelta [6] give explicit constructions for all 4-regular quadrangulations of the torus and Klein bottle. If $G$ is a quadrangulation of $S$ having no curvature vertices, then in fact $S$ must be the torus or Klein bottle. (See Proposition 2.1.)

\section{Basic properties of spherical quadrangulations}

Proposition 2.1 gives an arithmetic constraint on the quantities and degrees of curvature vertices in quadrangulations, and Corollary 2.2 is an immediate consequence. We use $\chi(S)$ to denote the Euler Characteristic of the surface $S$.

Proposition 2.1. If $G$ is a quadrangulation of closed surface $S$ with minimum degree 3 and $v_{i}$ vertices of degree $i$ then,

$$
v_{3}=4 \chi(S)+\sum_{i \geq 5}(i-4) v_{i} .
$$


Furthermore, if $\chi(S) \neq 0$, then there are curvature vertices.

Proof. If $G$ has $f$ faces and $e$ edges, then $\sum_{i} i v_{i}=2 e$. Also, $4 f=2 e$ and $\left(\sum_{i} v_{i}\right)-e+$ $f=\chi(S)$ which when combined together yield $4\left(\sum_{i} v_{i}\right)=4 \chi(S)+2 e$. Now subtracting we obtain $\sum_{i}(4-i) v_{i}=4 \chi(S)$ which yields our desired results.

Corollary 2.2. If $G$ is a spherical quadrangulation with $n$-fold rotational symmetry $(n \geq 3)$, minimum degree 3 , and having the least possible number of curvature vertices, then

- if $n=3$, then $G$ has eight vertices of degree 3 of which two are poles of the rotational symmetry;

- if $n=4$, then $G$ has 8 vertices of degree 3 and the two poles of the rotational symmetry are either both at vertices of degree 4 or both at the centers of faces; and

- if $n>4$, then $G$ has two vertices of degree $n$ and $2 n$ vertices of degree 3 where the two vertices of degree $n$ are the two poles of the rotational symmetry.

In [5], Graver and Hartung give a complete construction of spherical quadrangulations of the form $G=\mathcal{O}\left(H, H^{*}\right)$ where $H \cong H^{*}$ is a planar graph with four vertices of degree 3 and all other vertices of degree 4 . (They do not assume any rotational symmetry). Here, for $n=3$, we assume only that $G$ is a spherical quadrangulation with 3-fold rotational symmetry and discover structures not found in [5].

Given a vertex $v$ in a graph $G$ and a subgraph $H$ of $G$, the difference $d_{G}(v)-d_{H}(v)$ (that is, the degree of $v$ in $G$ minus the degree of $v$ in $H$ ) is called the codegree of $v$ with respect to $H$ and $G$. We say that $v$ is saturated by a subgraph $H$ when $v$ has codegree zero with respect to $H$ and $G$.

Consider a spherical quadrangulation $G$ with topological dual graph $G^{*}$. A collection $X$ of faces of $G$ corresponds to a collection of vertices $X^{*}$ of $G^{*}$. If the induced subgraph of $G^{*}$ on vertex set $X^{*}$ is connected, then the union of the faces in $X$ along their incident edges and vertices is called a face-connected subsurface. Let $F$ be the face-connected subsurface corresponding to $X$. The boundary of $F$, call it $\partial F$, is the collection of edges (and their endpoints) incident to exactly one face in $X$. Topologically speaking, a faceconnected subsurface $F$ is a sphere with holes (including the possibility of no holes) where, of course, if there is exactly one hole, then $F$ is topologically a disk. The boundary $\partial F$ is now an edge-disjoint union of cycles in $G$ bounding the holes of $F$. The total length of the union of boundary cycles is called the circumference or total circumference of $F$. An interior vertex of $F$ is a vertex not on $\partial F$ while a boundary vertex is a vertex on $\partial F$.

The distance between two vertices $u$ and $v$ in a graph $G$ is the length (edge length) of a shortest $u v$-path in $G$. Denote this distance by $\mathrm{d}_{G}(u, v)$. Of course, $\mathrm{d}_{G}(u, v)$ is even iff $u$ and $v$ are either both black or both white. Given a vertex $v$ in a spherical quadrangulation $G$, consider a face $F$ with white vertices $w_{1}$ and $w_{2}$ and black vertices $b_{1}$ and $b_{2}$. For any vertex $v$ in $G$, evidently, $\left|\mathrm{d}_{G}\left(v, w_{i}\right)-\mathrm{d}_{G}\left(v, b_{j}\right)\right|=1$ for each $i$ and $j \in\{1,2\}$. Additionally, the following three possibilities may occur for $F$ with respect to $v: \mathrm{d}_{G}\left(v, w_{1}\right)=\mathrm{d}_{G}\left(v, w_{2}\right)$ and $\left|\mathrm{d}_{G}\left(v, b_{1}\right)-\mathrm{d}_{G}\left(v, b_{2}\right)\right|=2 ;\left|\mathrm{d}_{G}\left(v, w_{1}\right)-\mathrm{d}_{G}\left(v, w_{2}\right)\right|=2$ and $\mathrm{d}_{G}\left(v, b_{1}\right)=\mathrm{d}_{G}\left(v, b_{2}\right)$; and $\mathrm{d}_{G}\left(v, w_{1}\right)=\mathrm{d}_{G}\left(v, w_{2}\right)$ and $\mathrm{d}_{G}\left(v, b_{1}\right)=\mathrm{d}_{G}\left(v, b_{2}\right)$.

Given a vertex $v$ and a face $f$ in a spherical quadrangulation $G$, let $u$ be a vertex on $f$ of smallest distance from $v$, say distance $t$. The vertices in cyclic ordering around $f$ now 
have distances $t, t+1, t+d, t+1$ from $v$ where $d \in\{0,2\}$. Given integer $k \geq 0$, define $X_{k}(v)$ to be the set of faces $f$ for which $t+1 \leq k$. The face-connected subsurface of $G$ given by $X_{k}(v)$ in Proposition 2.3 is called the $k$-ball centered at $v$ and is denoted $\mathrm{B}_{k}(v)$.

Proposition 2.3. The faces in $X_{k}(v)$ define a face-connected subsurface of $G$.

Proof. Certainly $X_{1}(v)$ is a face-connected subsurface. Using induction, assume that $X_{k-1}(v)$ is a face-connected subsurface and consider a face $f$ in $X_{k}(v)$ that is not in $X_{k-1}(v)$. We will complete the proof by showing that there is a facial path (that is, a path in $G^{*}$ ) from $f$ to a face in $X_{k-1}(v)$. Let $u$ be a vertex on $f$ whose distance from $v$ is the smallest and let $x_{1}$ and $x_{2}$ be the neighbors of $u$ on $f$. If we write $\mathrm{d}_{G}(v, u)=t$, then $\mathrm{d}_{G}\left(v, x_{1}\right)=\mathrm{d}_{G}\left(v, x_{2}\right)=t+1$. It must be that $t+1=k$ or else $f$ would be in $X_{k-1}(v)$.

Now consider the neighbors of $u$ in $G$, say $x_{1}, x_{2}, \ldots, x_{m}$. Since $\mathrm{d}_{G}(v, u)=k-1$, we get that $\mathrm{d}_{G}\left(v, x_{i}\right) \in\{k-2, k\}$ for each $i$ which implies that all faces of $G$ incident to $u$ are in $X_{k}(v)$. Now there must be some $i$ for which $\mathrm{d}_{G}\left(v, x_{i}\right)=k-2$ and so there is some face $f^{\prime}$ incident to $u$ which is in $X_{k-1}(v)$. The rotation of faces in $G$ around $u$ contains a path of adjacent faces from $f$ to $f^{\prime}$.

The reader can easily verify that Proposition 2.4 follows directly from definitions.

Proposition 2.4. If $v$ is a vertex in a spherical quadrangulation $G$ and $k \geq 1$, then the following hold.

(1) If $e$ is an edge of $G$ whose vertices have distances $t$ and $t+1$ from $v$ with $t+1 \leq k$, then both faces of $G$ incident to e are in $\mathrm{B}_{k}(v)$.

(2) If $u$ is a vertex of $G$ having distance $t \leq k$ from $v$, then $u$ is in $\mathrm{B}_{k}(v)$.

(3) If $f$ is a face of $\mathrm{B}_{k}(v)$ sharing an edge with $\partial \mathrm{B}_{k}(v)$, then the vertices of $f$ have distances $k-1, k, k+1, k$ from $v$; furthermore, an edge of $f$ on $\partial \mathrm{B}_{k}(v)$ has endpoints with distances $k$ and $k+1$ from $v$.

(4) If $f^{\prime}$ is a face that is not in $\mathrm{B}_{k}(v)$ and which shares an edge with $\partial \mathrm{B}_{k}(v)$, then the vertices of $f^{\prime}$ have distances $k, k+1, k+d, k+1$ from $v$ where $d \in\{0,2\}$.

(5) The vertices of a cycle $C$ on $\partial \mathrm{B}_{k}(v)$ have distance from $v$ alternating $k$ and $k+1$.

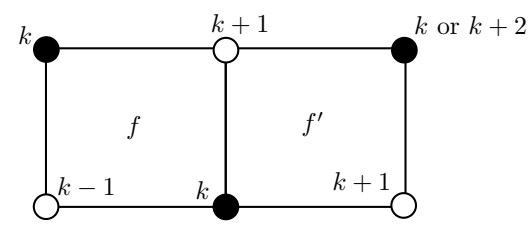

Figure 3: Proposition 2.4(4).

A standard $k$-disk with $n$-fold rotational symmetry around a fixed black vertex is constructed as follows. Consider the standard $\{4,4\}$ planar quadrangulation and designate one black vertex as an origin, and then label perpendicular $x$ - and $y$-axes. Consider the part of the quadrangulation in the first quadrant, with coordinate axes included, consisting of the union of the closed faces whose interiors are either beneath or intersecting the line 
$x+y=k$ (see Figure 4). Call this planar graph a $k$-wedge. Taking $n$ copies of a $k$-wedge, $W_{1}, \ldots, W_{n}$, identify the $x$-axis of $W_{i}$ with the $y$-axis of $W_{i+1}$ (subscript addition taken modulo $n$ ) with origin vertex identified to origin vertex to obtain the standard $k$-disk. In Figure 5 we show the standard 3-and 4-disks with a central vertex of degree $n=5$.

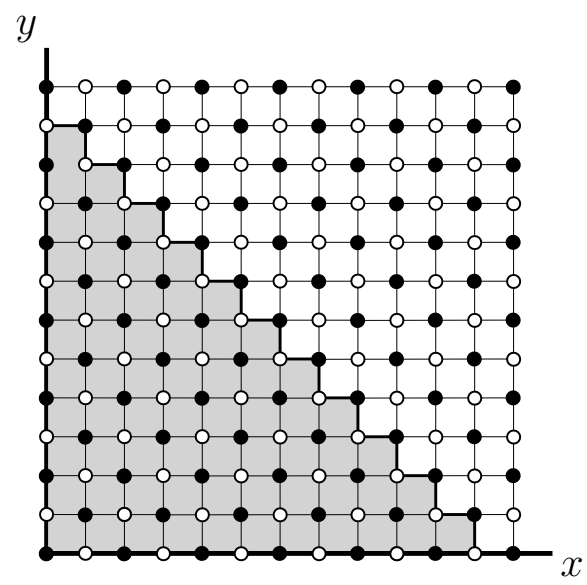

Figure 4: The 11-wedge.
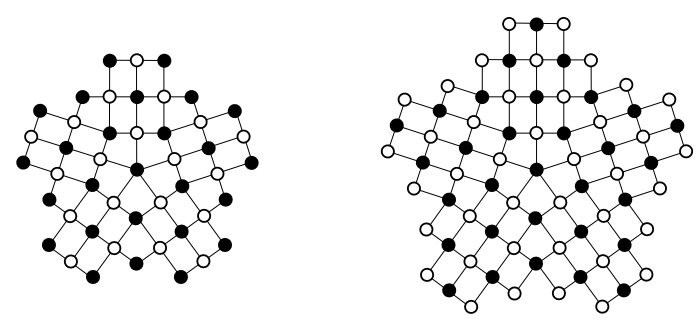

Figure 5: Standard 3- and 4-disks.

Consider a degree- 4 vertex in $G$ with incident edges $e_{1}, e_{2}, e_{3}, e_{4}$ in rotational order. We call each of the pairs $e_{1}, e_{3}$ and $e_{2}, e_{4}$ transverse. A path $P$ in a planar graph $G$ is said to be a transverse if each of its interior vertices has degree four in $G$ with consecutive edges on $P$ forming a transverse pair. In a standard $k$-disk, there are $n$ distinct transverse paths of length $k$ emanating from the origin (call it $p$ ) which we call the central rays. Note that every vertex of distance $k$ from $p$ in a standard $k$-disk $K$ has degree 4 in $K$ aside from the $n$ endpoints of the central rays which have degree 3 in $K$. The vertices of distance $k+1$ from $p$ in $K$ have degree 2 in $K$.

Proposition 2.5. The circumference of the standard $k$-disk is $2 n k$ and the number of faces in the standard $k$-disk is $n\left(\begin{array}{c}k+1 \\ 2\end{array}\right)$. 
Proof. The number of faces in the first quadrant of the coordinatized $\{4,4\}$ planar quadrangulation that intersect the line $x+y=k$ is $k$. Therefore the number of boundary edges of the $k$-wedge is $2 k$ and the number of faces in the $k$-wedge is a triangular sum. The result follows.

Proposition 2.6. If $G$ is a spherical quadrangulation and $\mathrm{B}_{k}(p)$ is a standard $k$-disk in $G$, then every vertex of distance $k$ from $p$ is on $\partial \mathrm{B}_{k}(p)$. Furthermore, any vertex of distance $k+1$ from $p$ is either on $\partial \mathrm{B}_{k}(p)$ or is a neighbor of some vertex of distance $k$ from $p$ on $\partial \mathrm{B}_{k}(p)$ that is not saturated by $\mathrm{B}_{k}(p)$.

Proof. Here $\partial \mathrm{B}_{k}(p)$ is a single cycle separating the sphere into regions $R_{1}$ and $R_{2}$ where without loss of generality $p$ is in $R_{1}$. Since $\mathrm{B}_{k}(p)$ is a standard $k$-disk, we know that every interior vertex of $\mathrm{B}_{k}(p)$ has distance less than $k$ from $p$. Given a vertex $v$ in $R_{2}$, a shortest $p v$-path in $G$ must pass through $\partial \mathrm{B}_{k}(p)$ and so $v$ has distance strictly larger than $k$. This implies our desired result.

Proposition 2.7. If $D$ is a disk in a spherical quadrangulation $G$ defined by a set of faces $X$ with $|X| \geq 2$, then there are two distinct faces $f_{1}, f_{2} \in X$ such that each $X-f_{i}$ defines a disk $D^{\prime}$ for which the intersection of the boundary of face $f_{i}$ with $\partial D^{\prime}$ is a path.

Proof. Consider a face $f \in X$ whose boundary shares an edge with $\partial D$, call this a boundary face. Using the fact that $D$ is a disk, the reader can confirm that the following are equivalent. (Here $G^{*}\left[Y^{*}\right]$ is the induced subgraph of $G^{*}$ on vertices $Y^{*}$ where $Y$ is a collection of faces in $G$.)

- The faces $X-f$ define a disk.

- The faces $X-f$ define a face-connected subsurface of $G$, that is, $G^{*}\left[X^{*}-f^{*}\right]$ is connected.

- The intersection of $f$ with $\partial D$ is a single path.

Assume by way of contradiction that $G^{*}\left[X^{*}-f^{*}\right]$ is disconnected for each boundary face $f \in X$. By disconnectedness, the degree of $f^{*}$ in $G^{*}\left[X^{*}\right]$ is not 1 ; furthermore, since $f$ is a boundary face, the degree of $f^{*}$ in $G^{*}\left[X^{*}\right]$ is either 2 or 3 . The number of connected components of $G^{*}\left[X^{*}-f^{*}\right]$ is two when the degree is 2 and is two or three when the degree is 3 . We get 3 connected components precisely when $f$ intersects $\partial D$ in three paths of lengths 1,0 , and 0 . Each connected component of $G^{*}\left[X^{*}-f^{*}\right]$ must contain a vertex corresponding to a boundary face of $D$.

Let $f \in X$ be a boundary face for which the induced subgraph of $G^{*}\left[X^{*}-f^{*}\right]$ contains a connected component on vertex set $C \subseteq X^{*}-f^{*}$ with $|C|$ as small as possible. Pick $f_{0}^{*} \in C$ that is a boundary face of $D$. By assumption, $G^{*}\left[X^{*}-f_{0}^{*}\right]$ is disconnected; however, by planarity, one of its connected components has vertex set which is a proper subset of $C$, a contradiction of minimality.

Given a face $f_{1} \in X$ such that $G^{*}\left[X^{*}-f_{1}^{*}\right]$ is connected, we will now find a face $f_{2} \neq f_{1}$ such that $G^{*}\left[X^{*}-f_{2}^{*}\right]$ is connected. Since $|X| \geq 2$, there must be boundary faces in $D$ other than $f_{1}$. By way of contradiction, assume that for every boundary face $f_{2} \neq f_{1}$ we have that $G^{*}\left[X^{*}-f_{2}^{*}\right]$ is disconnected. Pick $f_{2}$ such that $G^{*}\left[X^{*}-f_{2}^{*}\right]$ has a connected component on vertices $C$ with $f_{1}^{*} \notin C$ and $|C|$ as small as possible. Following the same argument as above, we will contradict the minimality of $C$. 
Proposition 2.8 is surely an expected outcome; however, there are subtleties that require verification. Proposition 2.8 provides a standard model for iteratively building up disks in G.

Proposition 2.8. Let $D$ be a disk in a spherical quadrangulation $G$ such that $D$ contains a black vertex $p$ having degree $n \geq 3$ in $G$ and all other vertices of $D$ have degree 4 in $G$. Then for $k$ large enough, $D$ is isomorphic to a face-connected subsurface of the standard $k$-disk with $n$-fold rotational symmetry around a black central vertex $p_{0}$. Furthermore, $p$ is identified with $p_{0}$.

Proof. Let $X$ be the collection of faces defining $D$. If $|X|=1$, then the result is clearly true. If $|X| \geq 2$, then by Proposition 2.7, there is an ordering $f_{1}, \ldots, f_{m}$ of the elements of $X$, such that $p$ is on $f_{1}$ and $X_{i}=\left\{f_{1}, \ldots, f_{i}\right\}$ defines a disk $D_{i}$ such that $f_{i+1}$ intersects $\partial D_{i}$ in a path. Inductively $D_{i}$ is a face-connected subsurface of a standard $k$-disk, $S_{k}$, for some large enough value of $k$. If $\partial D_{i}$ intersects $\partial S_{k}$, then increase $k$ by 2 so that $\partial D_{i}$ no longer intersects the boundary of the $k$-disk.

Let $P_{i}$ be the path of intersection of $f_{i+1}$ with $\partial D_{i}$ (the length of $P_{i}$ being 1,2 , or 3 ). Every internal vertex of $P_{i}$ must be saturated by $D_{i}$ as a subgraph of $G$ and each endpoint of $P_{i}$ is not saturated. Additionally, every vertex of $D$ other than $p$ has degree 4 in $G$, so the codegree of any vertex of $D_{i}$ is the same with respect to being a subgraph of $G$ or $S_{k}$. We now have that every internal vertex of $P_{i}$ is saturated by $D_{i}$ as a subgraph of $S_{k}$ and each endpoint of $P_{i}$ is not saturated. Thus $P_{i}$ is incident to a unique face $f^{\prime}$ of $S_{k}$ that is not in $D_{i}$. The face $f^{\prime}$ may now be identified with $f_{i+1}$ and we have $D_{i+1}$ as a subgraph of the standard $k$-disk $S_{k}$.

\section{The two constructions}

We will define two families of spherical quadrangulations, one defined with three independent parameters and the other with four. Each spherical quadrangulation is described as a net of $2 n$ congruent convex quadrilaterals with vertices on the 2-dimensional integer lattice in which two sides of the quadrilateral are perpendicular and of the same length. For lack of a more specific term, we will call such a quadrilateral a special integer quadrilateral.

\subsection{Three Parameters}

Choose positive even integer $a$, non-negative integer $s$, and $l \in\{0, \ldots, a-1\}$. Consider the special integer quadrilateral of Figure 6. By reflecting along the line $y=x$ we may assume that $l \in\left\{0, \ldots, \frac{a}{2}\right\}$

We assemble a net of $2 n$ such quadrilaterals as indicated in Figure 7 to obtain a spherical quadrangulation with $n$-fold rotational symmetry with poles at black vertices $p$ and $q$ and with all white vertices of degree 4 . Note that the arrangement of the quadrilaterals in this construction will always yield a quadrangulation with an order- 2 rotational symmetry which exchanges $p$ and $q$.

In Proposition 3.2 we characterize when the Three-Parameter Construction yields a spherical grid which is the overlay graph of a self-dual embedding. Proposition 3.1 gives us a necessary and sufficient condition for making this characterization. 


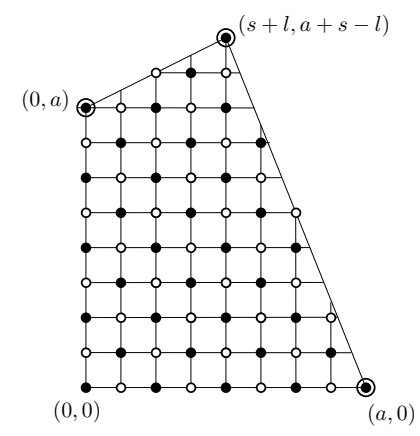

Figure 6: The Three-Parameter Construction with $(a, s, l)=(8,3,1)$.

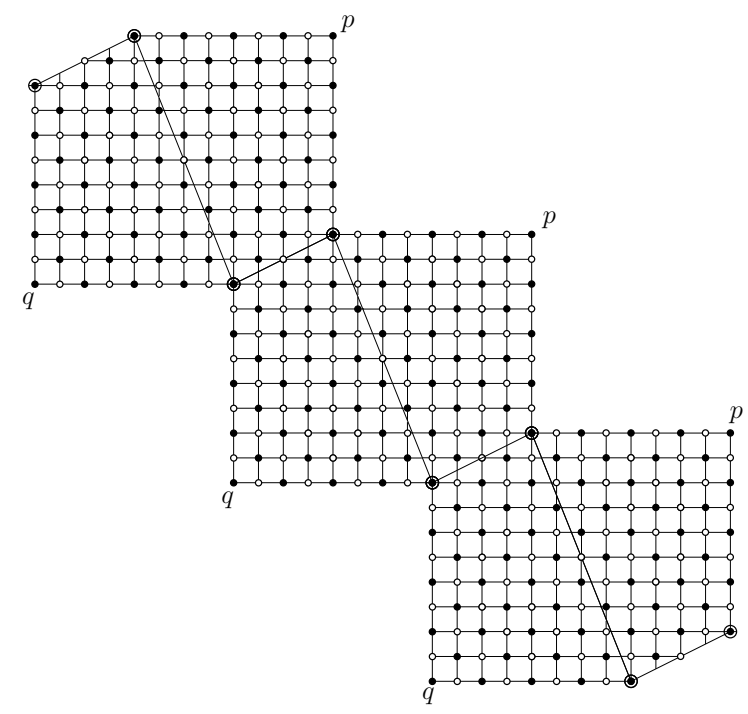

Figure 7: A net constructed from 6 copies of the quadrilateral in Figure 6.

Proposition 3.1. If $\mathcal{O}\left(H, H^{*}\right)$ has $n$-fold rotational symmetry with poles at black vertices, the minimum possible number of curvature vertices, and an additional symmetry that exchanges the poles, then $H \cong H^{*}$ if and only if one pole is in $H$ and the other in $H^{*}$.

Proof. Let $p$ and $q$ be the poles of the $n$-fold rotational symmetry of $\mathcal{O}\left(H, H^{*}\right)$. If one of $p$ and $q$ is in $H$ and the other in $H^{*}$, then because $\mathcal{R}\left(H, H^{*}\right)$ is bipartite, the symmetry which exchanges poles must exchange all of $H$ and $H^{*}$ and so is an isomorphism between $H$ and $H^{*}$.

Conversely, assume that $H \cong H^{*}$ and say that $H$ is red and $H^{*}$ is blue. As such, the rotational symmetry, which fixes $p$ and $q$, preserves the red and blue colors whereas the symmetry which exchanges $p$ and $q$ exchanges red and blue colors.

If $n=3$, then four of the degree-3 vertices are red and four are blue and $p$ and $q$ both have degree 3 . Furthermore, the six degree- 3 vertices other than $p$ and $q$ are therefore divided into two orbits under the rotational symmetry of three vertices each, one being red 
and the other blue. Therefore the fourth red degree- 3 vertex is one of $p$ and $q$ and the fourth blue degree- 3 vertex is the other.

If $n \geq 4$, then the rotational symmetry divides the $2 n$ degree- 3 vertices into two orbits of $n$ vertices each; one must be red and the other blue. Call these $O_{r}$ and $O_{b}$. Without loss of generality, say that $p$ is red. Thus the distance in $\mathcal{R}\left(H, H^{*}\right)$ from $p$ to the vertices in $O_{r}$ is even while the distance to the vertices in $O_{b}$ is odd. Now the symmetry which exchanges $p$ and $q$ must therefore exchange $O_{r}$ and $O_{b}$, more generally, exchange red and blue colors. Thus $p \in H$ and $q \in H^{*}$.

Proposition 3.2. A spherical quadrangulation constructed from the Three-Parameter Construction is the overlay graph of a self-dual graph if and only if $s$ and $l$ have different parities.

Proof. Let $G$ be a quadrangulation constructed using the Three-Parameter Construction and let $p$ and $q$ be the poles of the rotational symmetry. By Proposition 1.3, $G \cong \mathcal{O}\left(H, H^{*}\right)$ for some $H$. By Proposition 3.1, a necessary and sufficient condition for $H \cong H^{*}$ would without loss of generality be that $p \in H$ and $q \in H^{*}$. This is true if and only if the distance from $p$ to $q$ in $\mathcal{D}(G)$ is odd.

Consider one quadrilateral of the construction. In this quadrilateral, there is a path in the graph $\mathcal{D}(G)$, from $(0,0)$ to $(0, a)$ of length $a$. From $(0, a)$ to $(s+l, a+s-l)$, there is a path in $\mathcal{D}(G)$ of length $(s+l)+(s-l)=2 s$ when $s \equiv l \bmod 2$ and of length $(s+l-1)+(s-l-1)+1=2 s-1$ when $s \not \equiv l \bmod 2$. Thus there is a path from $p$ to $q$ in $\mathcal{D}(G)$ of length $2 a+2 s$ when $s \equiv l \bmod 2$ and of length $2 a+2 s-1$ when $s \not \equiv l$ $\bmod 2$, as required.

\subsection{Four parameters}

Choose positive integers $a$ and $b$ of the same parity. Assume that $a \geq b$. Choose nonnegative integers $h$ and $w$ of the same parity, not both zero, and which satisfy

$$
-\frac{h}{w} \leq \frac{b-a}{b+a} \quad \text { and } \quad-\frac{a}{b}(a-w) \leq b+h .
$$

(In the case that $w=0$ say that $-\frac{h}{w}=-\infty$.) Consider the special integer quadrilateral of Figure 8 .

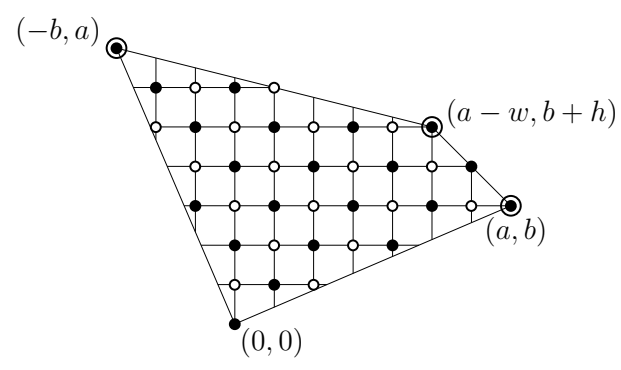

Figure 8: A special integer quadrilateral with $(a, b, h, w)=(7,3,2,2)$.

Given a choice of $a$ and $b$, the constraints placed on non-negative integers $h$ and $w$ guarantee that the quadrilateral defined will indeed be convex. The first inequality guarantees that the 
point $(a-w, b+h)$ lies above the line containing $(-b, a)$ and $(a, b)$. The second inequality guarantees that at $x=a-w$, the $y$-coordinate $b+h$ is greater than the $y$-coordinate of the line of slope $-\frac{b}{a}$. (See Figure 9.)

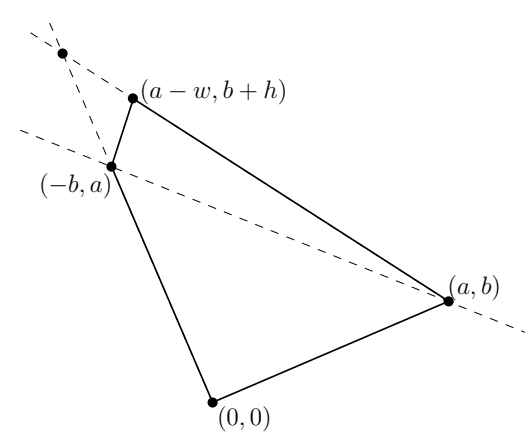

Figure 9: Constraints on $(a, b, h, w)$ guarantee convexity.

We assemble a net of $2 n$ such quadrilaterals as indicated in Figure 10 to obtain a spherical quadrangulation with $n$-fold rotational symmetry with poles at black vertices $p$ and $q$ and with every white vertex of degree 4 . As with the three-parameter construction, the fourparameter construction always yields a quadrangulation with an order- 2 rotational symmetry which exchanges $p$ and $q$.

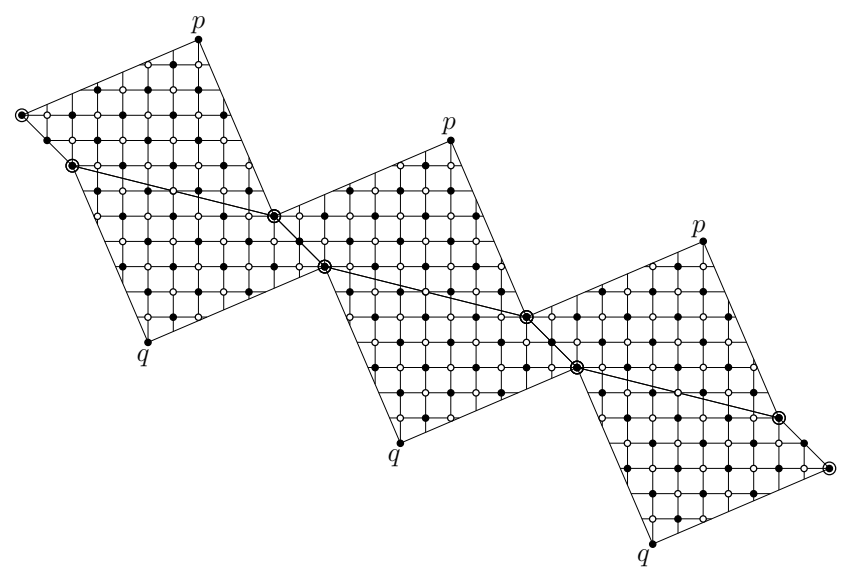

Figure 10: A net constructed from 6 copies of the quadrilateral in Figure 8.

Proposition 3.3. If $G$ is constructed from the four-parameter construction with parameters $(a, b, h, w)$, then $G$ is the overlay graph of a self-dual graph if and only if $h$ and $w$ are both odd.

Proof. Say $G$ is constructed using the Four-Parameter Construction and let $p$ and $q$ be the poles of the rotational symmetry. As in the proof of Proposition 3.2, $G \cong \mathcal{O}\left(H, H^{*}\right)$ for some $H$ (say $H$ is red and $H^{*}$ is blue) and a necessary and sufficient condition for $H \cong H^{*}$ would be that the distance from $p$ to $q$ in $\mathcal{D}(G)$ is odd. 
Consider one quadrilateral of the construction with $p$ at $(0,0)$. There is a path in $\mathcal{D}(G)$ from $p$ to the vertex at $(a, b)$ of length $a+b$ when $a$ and $b$ are both even and $a+b-1$ when $a$ and $b$ are both odd. Call this length $x$. There is a path in $\mathcal{D}(G)$ from $(a, b)$ to $(a-w, b+h)$ of length $w+h$ when $w$ and $h$ are both even and of length $w+h-1$ when $h$ and $w$ are both odd. Call this length $y$. Thus there is a $p q$-path in $\mathcal{D}(G)$ of length $2 x+y$ which is even when $h$ and $w$ are both even and is odd when $h$ and $w$ are both odd, as required.

\section{The two constructions are sufficient}

In this section we prove Theorem 1.1. So, throughout this section, let $n \geq 3$ be a fixed integer and $G$ a spherical quadrangulation satisfying the hypothesis of Theorem 1.1. Recall that making use of Proposition 1.3 allows this theorem to cover all spherical quadrangulations with minimum degree three and the minimum number of curvature vertices. Our first step is to prove Proposition 4.2, which separates the remainder of this proof into two distinct cases. In Section 4.3 we find that all graphs in the first case are given by the ThreeParameter Construction. In Section 4.4 we find that all graphs in the second case are given by the Four-Parameter Construction.

\subsection{Initial $k$-balls are standard disks}

Proposition 4.1. If every vertex of $\mathrm{B}_{k}(p)$ aside from $p$ has degree 4 in $G$, then $\mathrm{B}_{k+1}(p)$ is a standard $(k+1)$-disk.

Proof of Proposition 4.1. First we prove that $\mathrm{B}_{1}(p)$ is a standard 1-disk. The $n$ white neighbors of $p$ are all distinct because $G$ is simple. Now the only way in which $\mathrm{B}_{1}(p)$ is not a disk would be if the black vertices along the boundary walk are not all distinct. If, by way of contradiction, we assume that these black vertices are not all distinct, then rotational symmetry implies that there is only one black vertex on $\partial \mathrm{B}_{1}(p)$. This black vertex must therefore have degree $2 n$, a contradiction.

Inductively assume that the result holds up to some $k-1 \geq 0$ in $G$. Assume, by way of contradiction, that every vertex $v \neq p$ of $\mathrm{B}_{k}(p)$ has degree 4 in $G$ and yet $\mathrm{B}_{k+1}(p)$ is not a standard $(k+1)$-disk. Since every vertex $v \neq p$ of $\mathrm{B}_{k}(p)$ has degree 4 in $G$, we get that every vertex $v \neq p$ of $\mathrm{B}_{k-1}(p)$ has degree 4 in $G$ and so inductively $\mathrm{B}_{k}(p)$ is a standard $k$-disk.

By Proposition 2.4, every face $f$ that is not in $\mathrm{B}_{k}(p)$ but shares an edge $e$ with $\partial \mathrm{B}_{k}(p)$ is in $\mathrm{B}_{k+1}(p)$. Each vertex of distance $k$ from $p$ is in $\mathrm{B}_{k}(p)$ (again by Proposition 2.4) and so has degree 4 in $G$. Therefore each edge $e$ on $\partial \mathrm{B}_{k}(p)$ satisfies the following: if $e$ is incident to a central ray then the two edges of $f$ incident to $e$ are not on $\partial \mathrm{B}_{k}(p)$ and if $e$ is not incident to a central ray, then there are two consecutive edges of $f$ on $\partial \mathrm{B}_{k}(p)$ (see Figure 11).

The former type of face we will call a "radial" face and the latter a "notch" face. For a notch face there cannot be 3 edges of $f$ that are on $\partial \mathrm{B}_{k}(v)$ because this would either force a vertex on $\partial \mathrm{B}_{k}(p)$ to have degree less that 3 in $G$, a contradiction, or force two vertices on $\partial \mathrm{B}_{k}(p)$ to be identified, a contradiction because $\mathrm{B}_{k}(p)$ is a standard $k$-disk. In Case 1 assume that there is a radial face $f$ in $\mathrm{B}_{k+1}(p)$ having opposing edges that are both on $\partial \mathrm{B}_{k}(p)$. In Case 2, every radial face has exactly one of its edges on $\partial \mathrm{B}_{k}(p)$. 

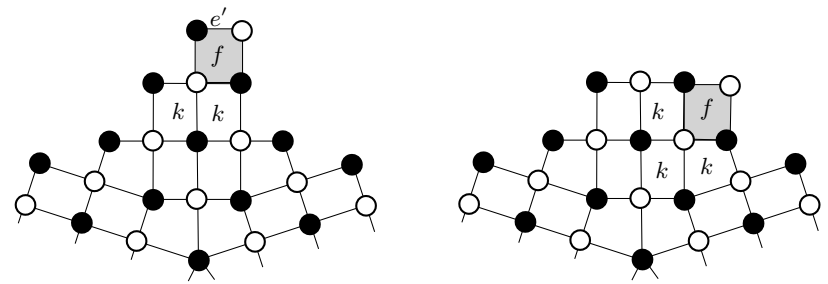

Figure 11: A radial face and a notch face.

Case 1: Let $e$ and $e^{\prime}$ be opposing edges of radial face $f$ that are both on $\partial \mathrm{B}_{k}(p)$. Since $e$ and $e^{\prime}$ are nonconsecutive on $f$, they must also be non-consecutive along $\partial \mathrm{B}_{k}(p)$ (see the left in Figure 11). Since $e$ and $e^{\prime}$ are the only edges of $f$ on $\partial \mathrm{B}_{k}(p)$, it must also be the case that $e^{\prime}$ is incident to a central ray of $\mathrm{B}_{k}(p)$ as well (which cannot be the same ray). Let $O$ be the orbit of $e$ under the $n$-fold rotational symmetry. (Note that $|O|=n$.) The black and white coloring of the vertices, along with orientability, forces edges $e$ and $e^{\prime}$ to both point in the same direction along the disk from their central rays, which implies that $e^{\prime} \in O$. Therefore the orbit of $f$ under the $n$-fold rotational symmetry does not have order $n$ and so $f$ contains a pseudofixed point in its interior, a contradiction of the fact that $p$ and $q$ are the only pseudofixed points of the rotational symmetry of the sphere.

Case 2: Let $B$ denote the face-connected subsurface consisting of $\mathrm{B}_{k}(p)$ along with the faces not in $\mathrm{B}_{k}(p)$ that share an edge with $\partial \mathrm{B}_{k}(p)$. Note that $B \subseteq \mathrm{B}_{k+1}(p)$. Take two faces $f$ and $f^{\prime}$ of $B$ that are not in $\mathrm{B}_{k}(p)$ which are consecutive with respect to their edges on $\partial \mathrm{B}_{k}(p)$. Because no vertex $v \neq p$ of $B \subseteq \mathrm{B}_{k+1}(p)$ has degree other than 4 , if one of $f$ and $f^{\prime}$ is a notch face then $f$ and $f^{\prime}$ share no edges in common, and if $f$ and $f^{\prime}$ are both radial faces then they must share one edge in common. Thus $B$ is obtained from a standard $(k+1)$-disk, call it $K$, after perhaps making identifications along $\partial K$. (See Figure 12.)

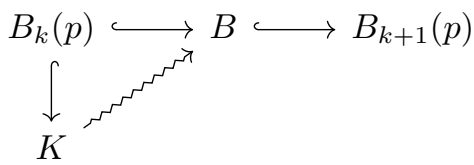

Figure 12: The relationships between $B, K, B_{k}(p)$ and $B_{k+1}(p)$.

Note that there can be no facial identifications in obtaining $B$ from $K$ because that forces the identified face to have two opposite edges on $\partial \mathrm{B}_{k}(p)$ which would put us back into Case 1. In Case 2.1 there are no identifications, in Case 2.2 there is an edge identification, and in Case 2.3 there are no edge identifications but there are vertex identifications.

Case 2.1: Here we have that $B=K$ is a standard $(k+1)$-disk. In this case, the vertices on the cycle $\partial B$ in $G$ must have distances from $p$ in $G$ alternating between $k+1$ and $k+2$. If $\mathrm{B}_{k+1}(p)=B$, then we are done. If not, then there is a face $f$ in $\mathrm{B}_{k+1}(p)$ that is not in $B$ and shares an edge with $\partial B$. Because $f$ is in $\mathrm{B}_{k+1}(p)$, the distances of the vertices on $f$ from $p$ must be $k+1, k+2, k+1, k$; however, $\partial B$ separates $p$ from $f$ in the sphere which means that no vertex on $f$ can have distance less than $k+1$ from $p$. 
Case 2.2: Let $e$ and $e^{\prime}$ be two edges on $\partial K$ that are identified in obtaining $B$ from $K$. If $e$ and $e^{\prime}$ are consecutive along $\partial K$ with $e$ having endpoints $u$ and $v$ and $e^{\prime}$ having endpoints $v$ and $w$, then the distances in $K$ from $p$ to $u, v$, and $w$ are either $k+1, k+2, k+1$ or $k+2, k+1$, and $k+2$. In the first case, the identification of $e$ and $e^{\prime}$ would create a vertex in $G$ of degree 1, a contradiction. In the second case, the identification of $e$ and $e^{\prime}$ would create either a vertex of degree 2 in $G$ (again a contradiction) or a vertex of degree 3 in $G$ that is in $B_{k}(p)$, a contradiction of our inductive hypothesis.

If $e$ and $e^{\prime}$ are in the same orbit under the rotational symmetry of $K$, then the interior of $e$ would contain a pseudofixed point of the rotational symmetry; however, $p$ and $q$ are the only pseudofixed points in $G$ of the rotational symmetry, a contradiction.

Now, given that $e$ and $e^{\prime}$ are not consecutive along $\partial K$ and not in the same orbit, rotational symmetry yields an orbit of $n$ distinct edges $e_{1}, \ldots, e_{n}$ identified to an orbit of $n$ distinct edges $e_{1}^{\prime}, \ldots, e_{n}^{\prime}$. Planarity of $G$ forces the cyclic ordering of these edges along $\partial K$ to be $e_{1}, e_{1}^{\prime}, \ldots, e_{n}, e_{n}^{\prime}$.

If either $e_{1}$ or $e_{1}^{\prime}$ (say $e_{1}$ ) is not incident to a central ray of $K$, then $e$ has an endpoint $v$ of degree 4 in $K$. Thus $v$ has distance $k+1$ from $p$ in $K$ and say without loss of generality that $v$ is black. The identification of $e_{1}$ and $e_{1}^{\prime}$ would force $v$ to have degree at least five in $B$ because the black endpoint of $e_{1}^{\prime}$ has degree at least 3 in $K$. This is impossible unless the vertex resulting from the identification of $e_{1}$ and $e_{1}^{\prime}$ is the other pole $q$; however, this implies that the corresponding endpoints of $e_{2}, \ldots, e_{n}$ and $e_{2}^{\prime}, \ldots, e_{n}^{\prime}$ are also the pole $q$. This is impossible because $q$ has degree $n$ in $G$ and such identifications would force $q$ to have degree at least $2 n$, which contradicts the fact that $G$ has maximum degree $n$.

If both $e_{1}$ and $e_{1}^{\prime}$ are incident to central rays, then these two orbits of edges account for all of the $2 n$ edges on $\partial K$ that are incident to the central rays. In Figure 13 the edges with the same numbers are identified and therefore, by the rotational symmetry, the endpoints of the central rays must be identified to the other pole of the rotational symmetry, call it $q$. Making these edge identifications results in a surface $K^{\prime}$ that is topologically a sphere with $n$ holes; that is, $\partial K^{\prime}$ consists of vertex-disjoint cycles $C_{1}, \ldots, C_{n}$. As discussed above, there can be no further edge identifications in going from $K^{\prime}$ to $B$. If there are vertex identifications in going from $K^{\prime}$ to $B$, then each identification is between two white degree-2 vertices on $\partial K^{\prime}$. These white vertices must be on the same cycle $C_{i}$. The reason for this is as follows. If vertex $x$ on $C_{i}$ is identified to vertex $y$ on $C_{j}$, then let $Q$ be a simple $x y$-path in $K^{\prime}$ whose interior avoids $\partial K^{\prime}$ (not necessarily a path in the graph). Now any cycle on $K^{\prime}$ which avoids its boundary (again, not necessarily a cycle in the graph itself) and separates $C_{i}$ from $C_{j}$ must transversely intersect $Q$ an odd number of times. Thus the spherical embedding $G$ would have two cycles drawn on it which intersect transversely an odd number of times, a contradiction.

Now say that two white vertices on $C_{i}$ (call them $x$ and $y$ ) are identified in going from $K^{\prime}$ to $B$. Note that the black vertices on $C_{i}$ all have degree 4 in $K^{\prime}$ and the white vertices on $C_{i}$ all have degree 2 in $K^{\prime}$ save for one which has degree 3 . Let $P_{i}$ be the $x y$-path on $C_{i}$ which avoids the degree- 3 white vertex. After identifying $x$ and $y$, facial boundaries of length four and saturated black vertices forces the identification of the adjacent pair of white vertices on $P_{i}$, and so on. These identifications will eventually result either in a white vertex being forced to have degree 2 in $G$ (a contradiction) or a face in $G$ being forced to have length 2 (again a contradiction).

Lastly, assuming there are no further vertex or edge identifications, we have $K^{\prime}=B$. Let $D_{i}$ be the disk in $G$ bounded by $C_{i}$ whose faces are not in $B$. The black vertices on 


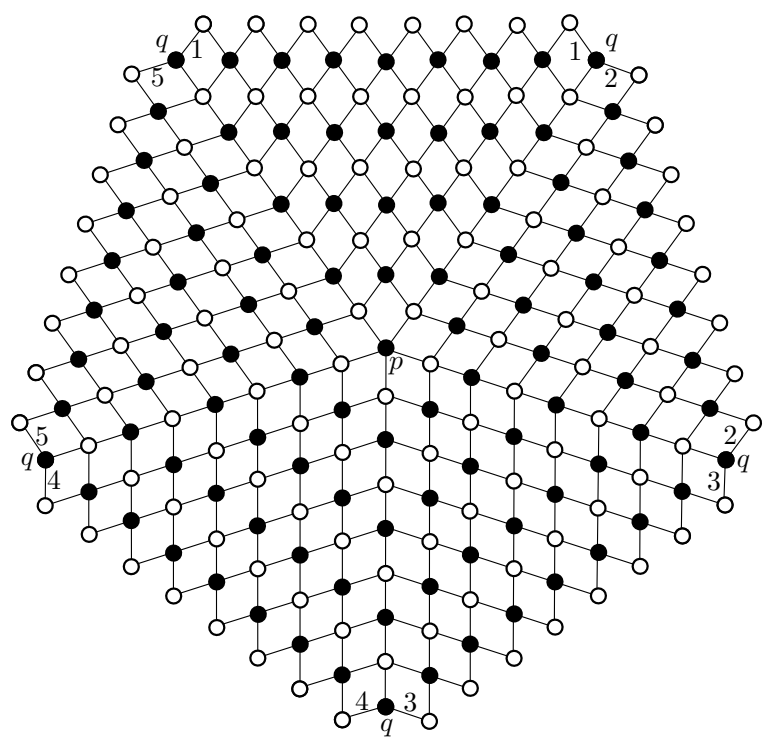

Figure 13: Edges with the same numbers are identified which then implies that the vertex $q$ is the other pole.

$C_{i}$ have degree 2 in $D_{i}$ and the white vertices on $C_{i}$ have degree 4 in $D_{i}$ save for one white vertex which has degree 3 in $D_{i}$. By rotational symmetry, there must be two black degree-3 vertices in the interior of $D_{i}$, with the remaining interior vertices having degree 4. This forces $D_{i}$ to have exactly three vertices of odd degree, a contradiction.

Case 2.3: If two identified vertices on $\partial K$ are in the same orbit under the rotational symmetry, then the resulting vertex will be pseudofixed and so the identified vertex is the pole $q$. However, $q$ will now be forced to have degree at least $2 n$, a contradiction. So now take an orbit of $n$ distinct vertices $v_{1}, \ldots, v_{n}$ on $\partial K$ that are pairwise identified to the $n$ distinct vertices $v_{1}^{\prime}, \ldots, v_{n}^{\prime}$ in going from $K$ to $B$. There can be no additional identifications among these vertices. Planarity now forces these $2 n$ vertices to have cyclic ordering $v_{1}, v_{1}^{\prime}, \ldots, v_{n}, v_{n}^{\prime}$ along $\partial K$. Thus the vertex $v_{1}=v_{1}^{\prime}$ in $G$ has degree 4 in $G$. So now if $K^{\prime}$ is obtained from $K$ by making these $n$ identifications only, then $K^{\prime}$ is obtained as shown in Figure 14 (for $n=5$ ).

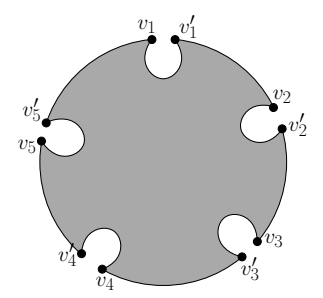

Figure 14: The surface $K^{\prime}$ from Case 2.3. 
Rechoose the identified vertex pairs $v_{1}, v_{1}^{\prime}, \ldots, v_{n}, v_{n}^{\prime}$ so that $v_{i}$ and $v_{i}^{\prime}$ are as close together along $\partial K$ as possible. Thus the orbit of $n$ holes in $K^{\prime}$ have boundaries that are cycles in $G$, say $C_{1}, \ldots, C_{n}$. Now either the endpoint of a central ray of $K^{\prime}$ is on $C_{1}$ and is adjacent to $v_{1}=v_{1}^{\prime}$ or not. Let these be Cases 2.3.1 and 2.3.2.

Case 2.3.1: Consider the disk $H$ in $G$ with $\partial H=C_{1}$ whose faces are not in $K$. By rotational symmetry either 0,1 , or 2 of the degree- 3 vertices of $G$ appear in $H$ and the pole $q$ does not appear in $H$. Assume for the moment that 0 of the degree-3 vertices of $G$ appear in $H$. Say that $C_{1}$ has length $2 m$. The degrees in $H$ of the vertices on $C_{1}$ are therefore $2,3,4,2,4,2, \ldots, 4,2$ in which the first degree- 2 vertex is $v_{1}=v_{1}^{\prime}$ and the degree- 3 vertex is the endpoint of the central ray of $K^{\prime}$. The remaining vertices of $H$ all have degree 4 . Thus the sum of the degrees of the vertices in $H$ is

$$
4 i+3+4(m-1)+2 m=4 i+6 m-1
$$

where $i$ is the number of interior vertices. So now if $\epsilon$ is the number of degree-3 vertices of $G$ appearing in $H$, then the sum of the degrees of the vertices in $H$ is $4 i+6 m-1-\epsilon$. Now if $e$ is the number of edges in $H$, we obtain

$$
4 i+6 m-1-\epsilon=2 e .
$$

If $f$ is the number of quadrilateral faces in $H$, then

$$
4 f+2 m=2 e .
$$

Now Euler's Formula implies that

$$
1=i+2 m-e+f=\frac{1}{4}(2 e+\epsilon+1-6 m)+2 m-e+\frac{1}{4}(2 e-2 m)=\frac{1}{4}(1+\epsilon) \leq \frac{3}{4},
$$

which is a contradiction.

Case 2.3.2: Let $u_{1}$ and $w_{1}$ be the neighbors of $v_{1}=v_{1}^{\prime}$ on $C_{1}$. Note that these three vertices all have degree 4 in $K^{\prime}$ and so have no edges extending into the interior of $H$. This forces these three vertices to be on the same quadrilateral face of $G$ and this face is in $H$. Because $v_{1}$ and $v_{1}^{\prime}$ are chosen to be as close together as possible along $\partial K$, it must be that $v_{1}$ and $v_{1}^{\prime}$ are at distance 4 apart along $\partial K$. Hence $C_{1}$ has length four and $H$ has only one face. Let $x$ be the fourth vertex of $C_{1}$. Since $u_{1}$ and $w_{1}$ both have degree 4 in $K^{\prime}$ and $K$, it must be that $x$ has degree 2 in $K^{\prime}$ which implies that $x$ also has degree 2 in $G$, a contradiction.

Proposition 4.2. If every vertex $v \neq p$ of $\mathrm{B}_{k-1}(p)$ has degree 4 in $G$ but there are curvature vertices of $G$ in $\mathrm{B}_{k}(p)-p$, then $\mathrm{B}_{k}(p)$ is a standard $k$-disk and the following hold.

(1) If $k$ is even, then the $n$ endpoints of the central rays of $\mathrm{B}_{k}(p)$ have degree 3 in $G$ and all other vertices of $\mathrm{B}_{k}(p)-p$ have degree 4 in $G$.

(2) If $k$ is odd, then there are either $n$ or $2 n$ degree-3 vertices of $G$ which have distance $k+1$ from $p$ on $\partial \mathrm{B}_{k}(p)$ and all other vertices of $\mathrm{B}_{k}(p)-p$ have degree 4 in $G$, including the endpoints of the central rays.

Proof. By Proposition 4.1, $\mathrm{B}_{k}(v)$ is a standard $k$-disk. By Proposition 2.4, the vertices of $\mathrm{B}_{k}(p)$ that are not in $\mathrm{B}_{k-1}(p)$ come in two types: those on $\partial \mathrm{B}_{k}(p)$ having distance $k+1$ 
from $p$ and the endpoints of the central rays of $\mathrm{B}_{k}(p)$, which have distance $k$ from $p$. If $k$ is odd, then because the curvature vertices of $G$ are black, $\mathrm{B}_{k}(p)$ is of Type (2). If $k$ is even, then because the curvature vertices of $G$ are black, $\mathrm{B}_{k}(p)$ is of Type (1) and there are either $n$ or $2 n$ such curvature vertices.

Proposition 4.2 gives us two cases for $G$. In Section 4.3 we will discuss the case for $G$ in Part (1) of Proposition 4.2 and in Section 4.4 the case for Part (2) of Proposition 4.2.

\subsection{Necklaces}

Take quadrilaterals $q_{1}, \ldots, q_{n}$ whose vertices are properly colored black and white. Label the black vertices of $q_{i}$ with $b_{i, 1}$ and $b_{i, 2}$. A diamond necklace of length $n$ with a black diagonal is the graph obtained from $q_{1}, \ldots, q_{n}$ by identifying $b_{i, 2}$ with $b_{i+1,1}$ for each $i \in\{1, \ldots, n\}$ where addition in subscripts is taken modulo $n$ so as to obtain a cyclic arrangement of these quadrilaterals. The top of Figure 15 shows a diamond necklace with a black diagonal. A diamond necklace of length $n$ with a white diagonal is defined similarly. When $t$ diamond necklaces of the same length with diagonals of alternating colors are stacked together as on the bottom of Figure 15, we obtain a straight thorax of thickness $t$.

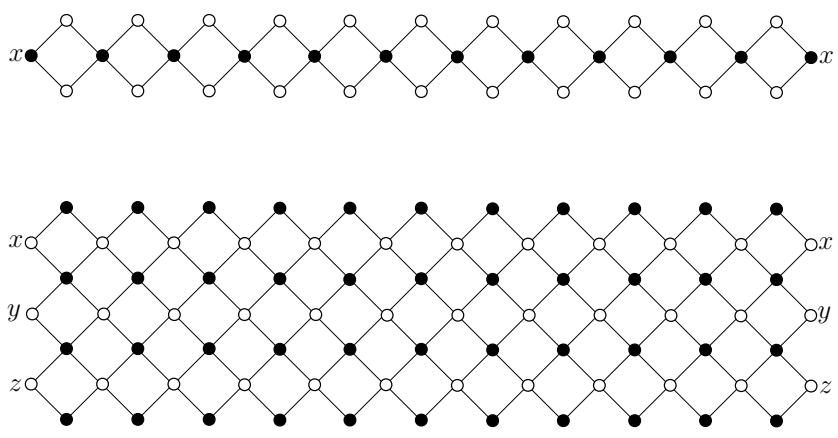

Figure 15: A diamond necklace with a black diagonal and a straight thorax of thickness 5 .

Consider a diamond necklace of length $n(k+1)$ for some $k \geq 1$ with black-diagonal vertices $b_{0}, \ldots, b_{n(k+1)-1}$ and choose a positive integer $1 \leq l \leq k$. If we embed our necklace in the plane with $b_{0}, \ldots, b_{n(k+1)-1}$ oriented in the clockwise direction, then there are well-defined inner and outer boundary cycles of length $2 n(k+1)$ each. For each vertex $i(k+1) \in\{0, k+1, \ldots,(n-1)(k+1)\}$ identify the two inner-boundary edges incident to $b_{i(k+1)}$ and identify the two outer-boundary edges incident to $b_{i(k+1)+l}$. The resulting graph with $n$-fold rotational symmetry is called a $(k, l)$-zig-zag necklace with a black diagonal. Note that the lengths of each of the two boundary cycles of the $(k, l)$-zigzag necklace is $2 n k$. A $(k, l)$-zig-zag necklace with a white diagonal is defined similarly. The graph in Figure 16 shows a portion of a $(k, 6)$-zig-zag necklace.

Any number of $(k, l)$-zig-zag necklaces with diagonals of alternating colors may be stacked as shown in Figure 17. 


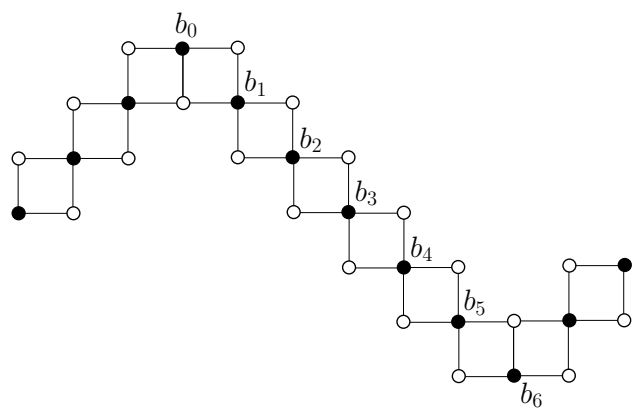

Figure 16: A portion of a $(k, 6)$-zig-zag necklace.

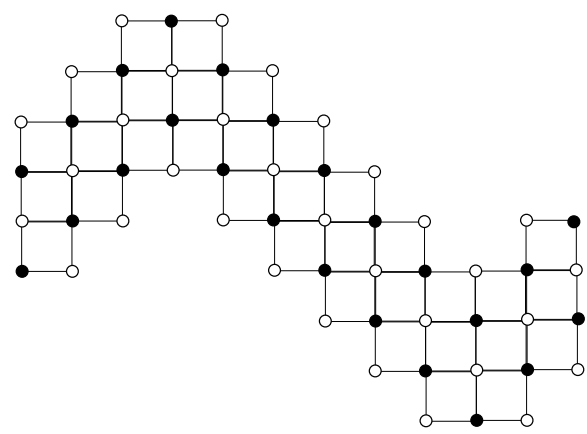

Figure 17: Stacking zig-zag necklaces.

\subsection{Curvature vertices on the ends of central rays}

Proposition 4.3. Let $\mathrm{B}_{k}(p)$ be a standard $k$-disk in $G$ as described in Proposition 4.2(1). If the only curvature vertices of $\mathrm{B}_{k+t}(p)$ are $p, v_{1}, \ldots, v_{n}$, then $\mathrm{B}_{k+t+1}(p)$ is a disk obtained from the standard disk $\mathrm{B}_{k}(p)$ by adding a straight thorax with thickness $t+1$. Furthermore, the circumferences of $\mathrm{B}_{k}(p)$ and $\mathrm{B}_{k+t}(p)$ are the same.

Proof. First we observe that the statement about the circumference of $\mathrm{B}_{k+t}(p)$ is evident by the structure of necklaces. We now proceed with the rest of the proof. Certainly for $t=0, \mathrm{~B}_{k+t}(p)$ is a disk obtained from the standard disk $\mathrm{B}_{k}(p)$ by adding a straight thorax with thickness $t=0$. So now assume that this same statement holds for some $t \geq 0$ and the only curvature vertices of $\mathrm{B}_{k+t}(p)$ are $p, v_{1}, \ldots, v_{n}$. Also as part of the induction hypothesis we include that $\partial \mathrm{B}_{k+t}(p)$ has vertices in one color class (those of distance $k+t$ from $p$ ) saturated by $\mathrm{B}_{k+t}(p)$ and vertices in the other color class (those of distance $k+t+1$ from $p$ ) having degree 2 in $\mathrm{B}_{k+t}(p)$.

Now consider $\mathrm{B}_{k+t+1}(p)$. Every face $f$ of $\mathrm{B}_{k+t+1}(p)$ that is not in $\mathrm{B}_{k+t}(p)$ yet shares an edge with $\partial \mathrm{B}_{k+t}(p)$ must share two consecutive edges with $\partial \mathrm{B}_{k+t}(p)$ because the vertices in one color class of $\partial \mathrm{B}_{k+t}(p)$ are saturated by $\mathrm{B}_{k+t}(p)$. Furthermore, since $\mathrm{B}_{k+t}(p)$ is a disk, $f$ cannot share three edges with $\partial \mathrm{B}_{k+t}(p)$ because if it did, then $f$ would have two vertices that are saturated by $\mathrm{B}_{k+t}(p)$ which would force $f$ to share all four of its edges with $\partial \mathrm{B}_{k+t}(p)$. Since $\partial \mathrm{B}_{k+t}(p)$ is a cycle, this would imply that the length of $\partial \mathrm{B}_{k+t}(p)$ is four; however, it must have length at least $2 n \geq 6$. 
Let $B$ be the face-connected subsurface obtained from $\mathrm{B}_{k+t}(p)$ by adding to it the faces of $\mathrm{B}_{k+t+1}(p)$ that share an edge with $\partial \mathrm{B}_{k+t}(p)$. We claim that $B$ is a disk. If $B$ is not a disk, then $B$ is obtained as follows. Let $B^{\prime}$ be the disk obtained from $\mathrm{B}_{k+t}(p)$ by adding to it a diamond necklace of faces. Now $B$ is obtained from $B^{\prime}$ by identifying edges or vertices on $\partial B^{\prime}$. Note that it is not possible to identify faces because $\partial \mathrm{B}_{k+t}(p)$ is a cycle.

It is not possible to identify two non-consecutive edges of $\partial B^{\prime}$ because any two such edges on $\partial B^{\prime}$ contain endpoints that are on $\partial \mathrm{B}_{k+t}(p)$, which is a disk, and therefore are distinct. Now suppose that $e_{1}$ and $e_{2}$ are consecutive edges on $\partial B^{\prime}$ whose common endpoint is $v$. The degree of $v$ in $B^{\prime}$ is either 2 or 4 . It is not possible that $v$ has degree 2 in $B^{\prime}$ because identifying $e_{1}$ and $e_{2}$ would then yield a vertex of degree $1 \mathrm{in} G$. It is not possible that $v$ has degree 4 in $B^{\prime}$ because identifying $e_{1}$ and $e_{2}$ would create a vertex of degree 3 in $\mathrm{B}_{k+t}(p)$ that is not among $p, v_{1}, \ldots, v_{n}$.

So it must be that $B$ is obtained from $B^{\prime}$ by identifications of vertices along $\partial B^{\prime}$. Note that the degree in $B^{\prime}$ of the vertices of $\partial B^{\prime}$ alternate between 2 and 4 where the degree- 2 vertices have distance $k+t+2$ from $p$ and the degree- 4 vertices have distance $k+t+1$ from $p$. Thus identification of any two degree- 2 vertices on $\partial B^{\prime}$ will then force the identification of another pair of degree- 2 vertices on $\partial B^{\prime}$. These identifications will continue until we force $G$ to contain either a facial cycle of length 2 (a contradiction) or a vertex of degree 2 (again a contradiction).

Thus $B^{\prime}=B$ is a disk and the vertices of $\partial B$ alternate with distances $k+t+1$ and $k+t+2$ from $p$. Thus $B=\mathrm{B}_{k+t+1}(p)$ because if there is a face $f$ in $\mathrm{B}_{k+t+1}(p)$ but not in $B$, its closest vertex to $p$ has distance at least $k+t+1$ and so this face is not in $\mathrm{B}_{k+t+1}(p)$, a contradiction.

Proposition 4.4. If $\mathrm{B}_{k+t}(p)$ is a disk in a spherical quadrangulation $G$ as given in Proposition $4.3, \mathrm{~B}_{k+t}(p)$ contains no curvature vertices of $G$ other than $p, v_{1}, \ldots, v_{n}$, but $\mathrm{B}_{k+t+1}(p)$ contains an additional curvature vertex of $G$, then $\mathrm{B}_{k+t+1}(p)$ is also a disk as given in Proposition 4.3, contains curvature vertices $u_{1}, \ldots, u_{n}$ on its boundary cycle, and each $u_{i}$ has distance $k+t+2$ from $p$.

Proof. This follows from Proposition 4.3 and the fact that the only vertices in $\mathrm{B}_{k+t+1}(p)$ that are not in $\mathrm{B}_{k+t}(p)$ are the outer vertices of the new diamond-necklace layer of the thorax.

Proposition 4.5. If $\mathrm{B}_{k+t}(p)$ is a disk as given in Proposition 4.4 which contains curvature vertices $p, v_{1}, \ldots, v_{n}$ in its interior and curvature vertices $u_{1}, \ldots u_{n}$ on its boundary, then $G$ is obtained from $\mathrm{B}_{k+t}(p)$ by identifying $\partial \mathrm{B}_{k+t}(p)$ with the boundary of a standard $k$-disk $D$ such that $u_{1}, \ldots, u_{n}$ are identified with the endpoints of the central rays of $D$.

Proof. By Proposition 4.4, each $u_{i}$ has distance $k+t+1$ from $p$. This implies that $k+t+1$ is even, and since $k$ is even, we must have that $t$ is odd.

Say that $l$ is the smallest distance in $G$ from the pole $q$ to any vertex on $\partial B_{k+t}(p)$ and let $u$ be such a vertex. It must be that $\mathrm{d}(u, p)=k+t+1$ rather than $k+t$, because the vertices of $\partial B_{k+t}(p)$ of distance $k+t$ from $p$ are saturated by $B_{k+t}(p)$ and so any path from $q$ to one of these vertices of distance $k+t$ from $p$ must go through the vertices of distance $k+t+1$ from $p$. Therefore $u$ is black and has degree 2 in $B_{k+t}(p)$. Also, since $u$ is black, $l$ must be even.

First suppose that $u$ can be chosen to be in $\mathrm{B}_{l-1}(q)$. The intersection of $\mathrm{B}_{l-1}(q)$ and $\mathrm{B}_{k+t}(p)$ may only consist of a collection of black vertices because the white vertices of 
$\mathrm{B}_{l-1}(q)$ have distance at most $l-1$ from $q$. Therefore $\mathrm{B}_{l-2}(q)$ contains no curvature vertices aside from $q$ and Proposition 4.1 implies that $\mathrm{B}_{l-1}(q)$ is a standard $(l-1)$-disk. Thus $u$ has degree 2 in $\mathrm{B}_{l-1}(q)$ and must have degree 4 in $G$. The neighbor $w_{p}$ of $u$ on $\partial \mathrm{B}_{k+t}(p)$ in the direction of rotation is saturated by $\mathrm{B}_{k+t}(p)$ and the neighbor $w_{q}$ of $u$ on $\partial \mathrm{B}_{l-1}(q)$ in the rotational direction is either saturated or, alternatively, is the endpoint of a central ray and, because it is white, has codegree 1 with respect to $\mathrm{B}_{l-1}(q)$. Let $u_{p}$ be the next vertex in rotational order along $\partial \mathrm{B}_{k+t}(p)$ and let $u_{q}$ be the next vertex in rotational order along $\partial \mathrm{B}_{l-1}(q)$. If $w_{q}$ is saturated by $\mathrm{B}_{l-1}(q)$, then because every face of $G$ has length four we must have that $u_{p}=u_{q}$ and this vertex has degree 4 in $G$. (See the left configuration in Figure 18). If $w_{q}$ is the end of a central ray of $\mathrm{B}_{l-1}(q)$ (which has codegree 1 ), then again, the fact all faces have length four implies that $u_{p}$ is adjacent to $w_{q}$ and so $u_{p}$ is a curvature vertex of $G$ and $u_{q}=u_{p}^{\prime}$ where $u_{p}^{\prime}$ is the next black vertex in the rotational direction on $\partial \mathrm{B}_{k+t}(p)$. (See the right configuration in Figure 18).
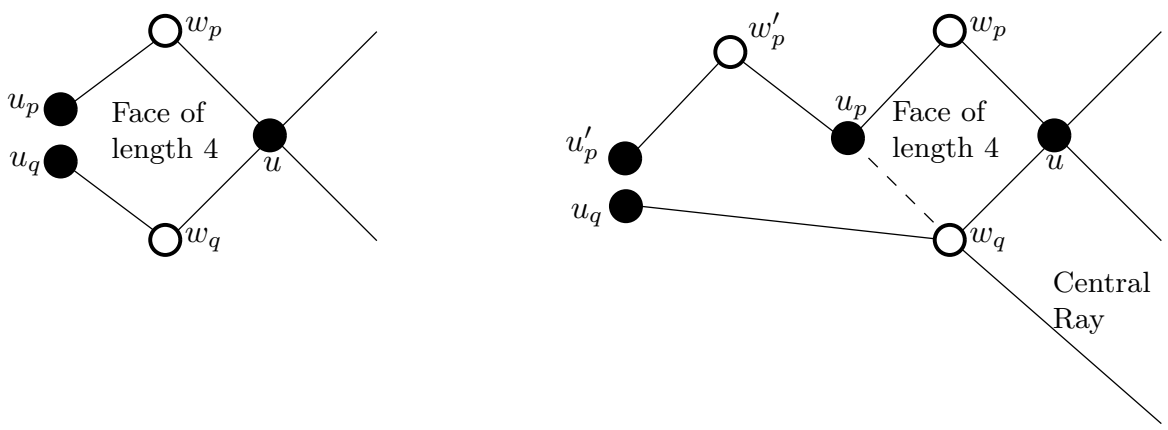

Figure 18: Forced vertex identifications on the boundaries of $\mathrm{B}_{k+1}(p)$ and a standard $k$ disk.

This process of identifying vertices and adjacencies continues all the way around $\partial \mathrm{B}_{k+t}(p)$ and $\partial \mathrm{B}_{l-1}(q)$ so that the black vertices on $\partial \mathrm{B}_{k+t}(p)$ correspond to the black vertices and endpoints of the central rays of $\mathrm{B}_{l-1}(q)$. Therefore $l=k$ and $G$ is obtained as stated in the proposition.

Next suppose that $u$ cannot be chosen to be in $\mathrm{B}_{l-1}(q)$. Proposition 2.6 implies that $u$ is the endpoint of a central ray of $B_{l}(q)$. Let $w$ be this endpoint of the central ray of $\mathrm{B}_{l-1}(q)$ that is adjacent to $u$. Thus $u$ has codegree 1 or 2 with respect to $B_{k+t}(p)$. In either case there is a face $f$ incident to the $u w$-edge that contains an edge $w b_{1}$ of $\partial \mathrm{B}_{l-1}(q)$ and an edge $u w_{1}$ of $\partial \mathrm{B}_{k+t}(p)$. So now a fourth edge for $f$ would be $w_{1} b_{1}$; however, $w_{1}$ is saturated by $\mathrm{B}_{k+t}(p)$ and $b_{1} \notin \mathrm{B}_{k+t}(p)$, a contradiction (see Figure 19).

Theorem 4.6. The graph described in Proposition 4.5 is given by the Three-Parameter Construction.

Proof. Consider the part of $\mathrm{B}_{k}(p)$ between two consecutive central rays, call it $W_{k}$. Let $o_{1}$ and $o_{2}$ be the curvature vertices on the central rays of $W_{k}$ which have distance $k$ from $p$. Consider the black diagonal line $D$ in $W_{k}$ from $o_{1}$ to $o_{2}$. Now let $W$ be the portion of $\mathrm{B}_{k+t}(p)$ consisting of $W_{k}$ along with the faces between the black diagonals emanating from $o_{1}$ and $o_{2}$ which are perpendicular to $D$. Let $o$ be the curvature vertex in $W$ which 


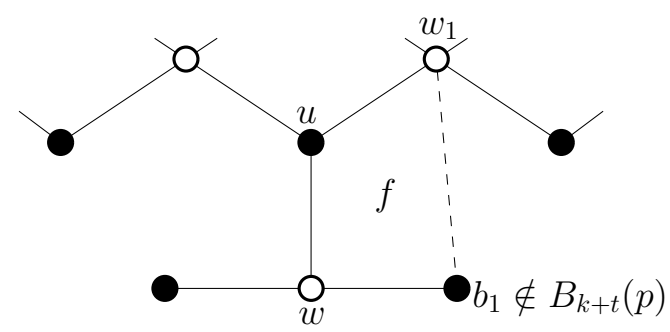

Figure 19: Final contradiction in the proof of Proposition 4.5.

has distance $k+t$ from $p$. As shown in Figure 20, there is a special integer quadrilateral for the Three-Parameter Construction contained within $W$.

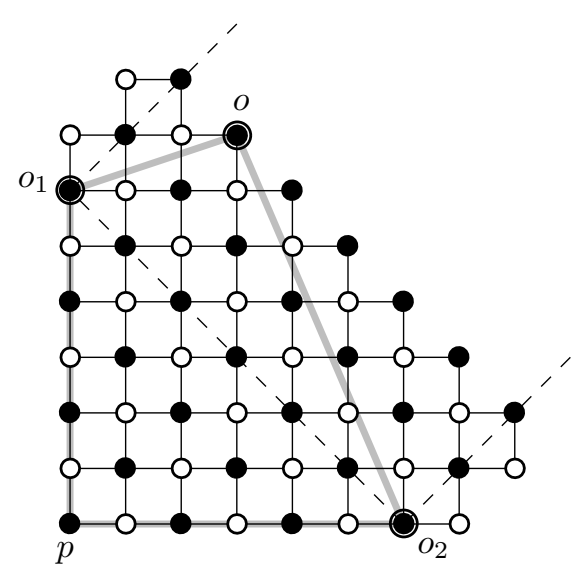

Figure 20: A special integer quadrilateral within a single wedge.

By inspection, the spherical quadrangulation constructed by the special integer quadrilateral from Figure 20 contains $B_{k+t}(p)$ with curvature vertices positioned as shown. By Proposition 4.5, there is only one spherical quadrangulation which contains $\mathrm{B}_{k+t}(p)$ with curvature vertices in a given position. Thus $G$ is given by the Three-Parameter Construction.

\subsection{Curvature vertices off the central rays}

Let $\mathrm{B}_{k}(p)$ be a standard $k$-disk in $G$ as described in Proposition 4.2(2). The disk $\mathrm{B}_{k}(p)$ contains at least one orbit of $n$ degree- 3 curvature vertices. Let $t \geq 0$ be the smallest integer for which $\mathrm{B}_{k+t}(p)$ contains both orbits of $n$ degree- 3 curvature vertices. In Proposition 4.8 we describe three possible structures for $\mathrm{B}_{k+t}(p)$. Finally, we show that each structure is given by the Four-Parameter Construction. 


\subsubsection{Two new types of disk}

Note that the length of $\partial \mathrm{B}_{k}(p)$ is $2 n k$. The black vertices along $\partial \mathrm{B}_{k}(p)$ have degree 2 in $\mathrm{B}_{k}(p)$ and the white vertices along $\partial \mathrm{B}_{k}(p)$ have degree 4 in $\mathrm{B}_{k}(p)$ save for the endpoints of the central rays which have degree 3 in $\mathrm{B}_{k}(p)$. Consider vertices $w_{1}, v_{1}, \ldots, w_{n}, v_{n}$ in clockwise order around $\partial \mathrm{B}_{k}(p)$ in which $w_{1}, \ldots, w_{n}$ are the ends of the central rays and $v_{1}, \ldots, v_{n}$ is one orbit of black vertices on $\partial \mathrm{B}_{k}(p)$. Say that the distance from $w_{i}$ to $v_{i}$ along $\partial \mathrm{B}_{k}(p)$ is $2 l-1$. Let $T$ be a $t$-layered stack of $(k, l)$-zig-zag necklaces. Note that $\partial T$ consists of two cycles. Let $\partial_{\text {in }} T$ be the inner cycle of $\partial T$ and say that the necklace along $\partial_{\text {in }} T$ has a black diagonal and label these black vertices as $b_{0}, \ldots, b_{n(k+1)-1}$. Note that $b_{j}$ for $j$ not divisible by $k+1$ appears on $\partial_{\text {in }} T$ and has degree four in $T$ except when $j=i(k+1)+l$, in which case $b_{j}$ has degree 3 in $T$. Also, the white vertices on $\partial_{\text {in }} T$ all have degree 2 in $T$ save for the white vertices on $\partial_{\text {in }} T$ adjacent to $b_{i(k+1)}$ 's, which have degree 3 in $T$. Thus we can identify $\partial \mathrm{B}_{k}(p)$ with $\partial_{\text {in }} T$ so that $v_{i}$ is identified with $b_{i(k+1)+l}$ and $w_{i}$ is adjacent to $b_{i(k+1)}$. We call the resulting disk $Z_{k, l, t}(p)$. Our discussion assumes that $t \geq 1$, but as a convention we can define $Z_{k, l, 0}(p)$ to be the standard $k$-disk with $l$ defined by either one of the two orbits of degree- 3 curvature vertices on $\partial \mathrm{B}_{k}(p)$. Note that $\mathrm{Z}_{k, l, t}(p)$ is a $(k+t)$-ball centered at $p$ and every vertex $v \neq p$ in the interior of $Z_{k, l, t}(p)$ has degree 4 in $Z_{k, l, t}(p)$ save for $v_{1}, \ldots, v_{n}$ which all have degree 3 . Figure 21 depicts $\mathrm{Z}_{5,2,3}(p)$ for $n=5$ (ignore the shading in the outer faces for the moment).

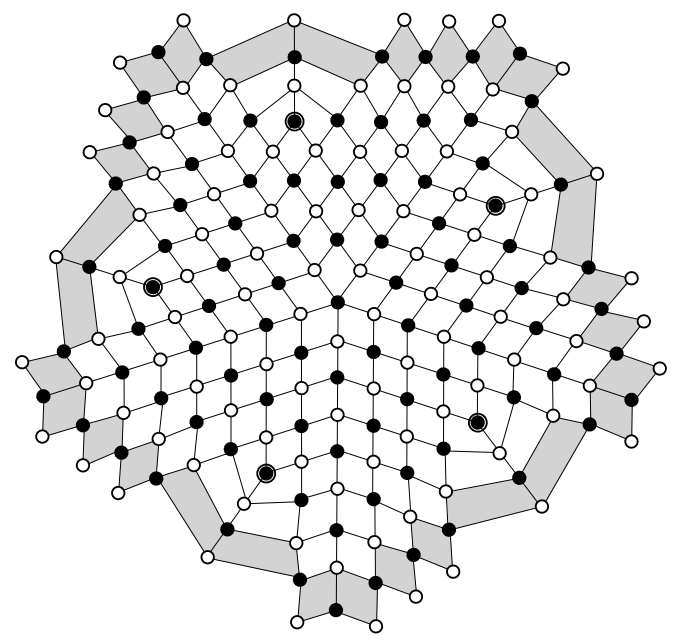

Figure 21: The disk $Z_{5,2,3}(p)$. If the shaded faces are removed, then the remaining faces define $\mathbf{Z}_{5,2,2}(p)$.

Now for $t \geq 2$ that is even we define a disk $\widehat{Z}_{k, l, t}(p)$ from $Z_{k, l, t}(p)$. Say that $v_{i}^{\prime}$ is the black vertex on $\partial \mathrm{Z}_{k, l, t}(p)$ that is on the transverse path from $v_{i}$ emanating outwards from $\mathrm{B}_{k}(p)$. (Call this transverse path from $v_{i}$ a curvature ray.) Also, say that $w_{i}^{\prime}$ is the endpoint of the central ray of $Z_{k, l, t}(p)$ that contains $w_{i}$. Now since $t$ is even, the black vertices on $\partial \mathrm{Z}_{k, l, t}(p)$ all have degree 2 except for $v_{1}^{\prime}, \ldots, v_{n}^{\prime}$, which have degree 3 in $\mathrm{Z}_{k, l, t}(p)$. Let $l^{\prime}=\min \{l-1, k-l\}$. Label the $l^{\prime}$ black vertices on $\partial Z_{k, l, t}(p)$ in the clockwise direction from $v_{i}^{\prime}$ with $1,2, \ldots, l^{\prime}$ and do the same for the $l^{\prime}$ black vertices on $\partial Z_{k, l, t}(p)$ 
in the counter-clockwise direction from $v_{i}^{\prime}$ (see the left-hand configuration in Figure 22 in which $k=7, l=3, t=2$, and $l^{\prime}=l-1=2$ ).
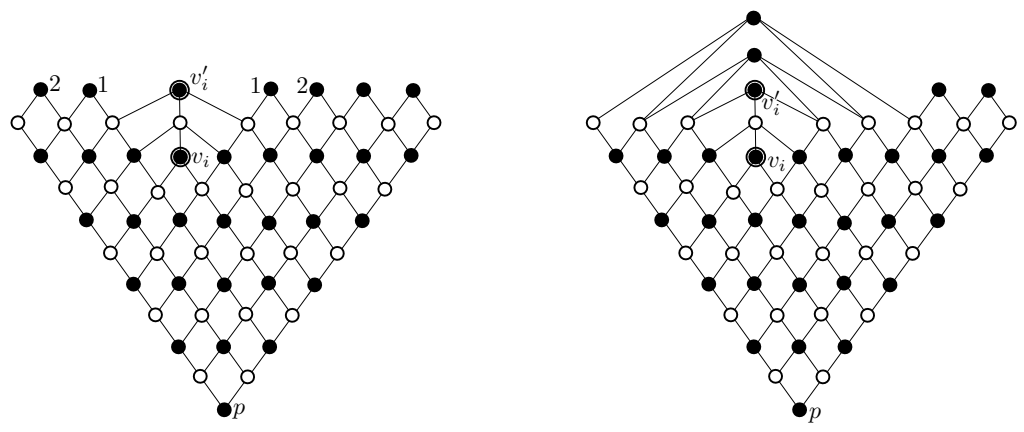

Figure 22: Constructing $\widehat{Z}_{k, l, t}(p)$ with $k=7, l=3, t=2$, and $l^{\prime}=l-1=2$.

Now identify the black vertices having the same labels as shown on the right in Figure 22. Repeat these identifications for each $i$. The resulting disk is $\widehat{Z}_{k, l, t}(p)$. Note that $\widehat{Z}_{k, l, t}(p)$ is a $(k+t)$-ball centered at $p$ and that every vertex $v \neq p$ in the interior of $\widehat{Z}_{k, l, t}(p)$ has degree 4 in $\widehat{Z}_{k, l, t}(p)$ save for $v_{1}, \ldots, v_{n}, v_{1}^{\prime}, \ldots, v_{n}^{\prime}$ which all have degree 3 .

\subsubsection{The three structures}

Proposition 4.7. Let $\mathrm{B}_{k}(p)$ be a standard $k$-disk in $G$ with $k$ odd and with exactly $n$ degree3 vertices $v_{1}, \ldots, v_{n}$ of $G$ appearing on $\partial \mathrm{B}_{k}(p)$. For each $t \geq 0$, if the only curvature vertices in $\mathrm{B}_{k+t-1}(p)$ are among $p, v_{1}, \ldots, v_{n}$, then $\mathrm{B}_{k+t}(p)$ is either $\mathrm{Z}_{k, l, t}(p)$ or $\widehat{Z}_{k, l, t}(p)$ where $l$ is specified by the position of $v_{1}, \ldots, v_{n}$ on $\partial \mathrm{B}_{k}(p)$.

Proof. The proof will be by induction on $t$ where the case for $t=0$ is given by Proposition 4.1. Assuming for some $t \geq 1$ that the only curvature vertices in $\mathrm{B}_{k+t-1}(p)$ are among $p, v_{1}, \ldots, v_{n}$ we now consider $\mathrm{B}_{k+t}(p)$. For $t=1$, we already know that $\mathrm{B}_{k+t-1}(p)=\mathrm{B}_{k}(p)$ is a standard $k$-disk which is also $\mathrm{Z}_{k, l, 0}(p)$. For $t \geq 2$, the induction hypothesis assumes that $\mathrm{B}_{k+t-1}(p)$ is either $Z_{k, l, t-1}(p)$ or $\widehat{Z}_{k, l, t-1}(p)$. However, while the only curvature vertices of $\mathrm{B}_{k+t-1}(p)$ are among $p, v_{1}, \ldots, v_{n}$, in fact, $\widehat{Z}_{k, l, t-1}(p)$ contains more curvature vertices than this. Hence $\mathrm{B}_{k+t-1}(p)=\mathrm{Z}_{k, l, t-1}(p)$.

By Proposition 2.4 every face of $G$ not in $\mathrm{B}_{k+t-1}(p)$ but sharing an edge with $\partial \mathrm{B}_{k+t-1}(p)$ is in $\mathrm{B}_{k+t}(p)$. Consider the face-connected subsurface $B \subseteq \mathrm{B}_{k+t}(p)$ consisting of $\mathrm{B}_{k+t-1}(p)$ along with the faces not in $\mathrm{B}_{k+t-1}(p)$ but sharing an edge with $\partial \mathrm{B}_{k+t-1}(p)$. We will show that $B=\mathrm{Z}_{k, l, t}(p)$ or $B=\widehat{Z}_{k, l, t}(p)$ and that $B=\mathrm{B}_{k+t}(p)$.

Given an edge $e$ of $\partial \mathrm{B}_{k+t-1}(p)=\partial \mathrm{Z}_{k, l, t-1}(p)$, let $f_{e}$ be the face of $B$ that is not in $\mathrm{B}_{k+t-1}(p)$ and is incident to $e$. For comparison as a standard model, consider the disk $\mathrm{Z}_{k, l, t}(p)$ (separate from $G$ ) whose subdisk $\mathrm{Z}_{k, l, t-1}(p)$ is identified with $\mathrm{B}_{k+t-1}(p)$ in $G$. Let $f_{e}^{\prime}$ be the face of $\mathrm{Z}_{k, l, t}(p)$ that is not in $\mathrm{B}_{k+t-1}(p)=\mathrm{Z}_{k, l, t-1}(p)$ and is incident to $e$. If $f_{e}$ (or $f_{e}^{\prime}$ ) is incident to a central ray, then call $f_{e}$ (or $f_{e}^{\prime}$ ) a radial face; otherwise, call $f_{e}$ (or $f_{e}^{\prime}$ ) a notch face. Note that $f_{e_{1}}^{\prime}=f_{e_{2}}^{\prime}$ if and only if $f_{e_{1}}^{\prime}$ is a notch face with $e_{1}$ and $e_{2}$ both incident to a common vertex that is saturated with respect to $\mathrm{B}_{k+t-1}(p)$ and $G$ (see 
Figure 21). We must show that the corresponding necessary and sufficient condition holds for $f_{e_{1}}=f_{e_{2}}$.

If $f_{e}$ is a notch face, then $f_{e}$ shares two consecutive edges (say $e_{1}$ and $e_{2}$ ) with $\partial \mathrm{B}_{k+t-1}(p)$ where the common endpoint of $e_{1}$ and $e_{2}$, call it $v_{12}$, is a vertex saturated by $\mathrm{B}_{k+t-1}(p)$ in $G$. It cannot be that a third edge of $f_{e}$ is on $\partial \mathrm{B}_{k+t-1}(p)$ because such an edge would have to be consecutive with $e_{1}$ or $e_{2}$ on the cycle $\partial \mathrm{B}_{k+t-1}(p)$, whereas the two endpoints of $e_{1}$ and $e_{2}$ other than $v_{12}$ have codegree 1 or 2 with respect to $\mathrm{B}_{k+t-1}(p)$.

If $f_{e}$ is a radial face of $B$, then denote the edges of $f_{e}$ by $e_{1}, e_{2}, e_{3}, e_{4}$ in rotational order along $f_{e}$. Assuming that $e_{1}$ is on $\partial \mathrm{B}_{k+t-1}(p)$ and is incident to a central ray of $\mathrm{B}_{k+t-1}(p)$ (call it $r$ ) we get that each endpoint of $e_{1}$ has positive codegree with respect to $\mathrm{B}_{k+t-1}(p)$. Thus $e_{2}$ and $e_{4}$ are not on the cycle $\partial \mathrm{B}_{k+t-1}(p)$. Without loss of generality assume that edge $e_{2}$ is the transverse continuation of $r$. Assume by way of contradiction that $e_{3}$ is on $\partial \mathrm{B}_{k+t-1}(p)$. Since $e_{2}$ and $e_{4}$ are not on $\partial \mathrm{B}_{k+t-1}(p)$, we again must have that $e_{3}$ is also incident to a central ray of $\mathrm{B}_{k+t-1}(p)$, call it $r^{\prime}$. Note that $r \neq r^{\prime}$ because $r=r^{\prime}$ would imply that $G$ is not simple, a contradiction. Now either $e_{1}$ and $e_{3}$ are in the same orbit under the rotational symmetry or not. If so, then the orbit of $f_{e}$ under the rotational symmetry consists of $n / 2$ faces and so there is a pseudofixed point in the interior of $f_{e}$, a contradiction. If not, then when adding $f_{e}$ to $\mathrm{B}_{k+t-1}(p)$, the black and white bipartition forces there to be a half twist which creates a Möbius band in $G$, a contradiction.

The previous two paragraphs show that $f_{e} \mapsto f_{e}^{\prime}$ is a one to one correspondence between the faces of $B$ that are not in $\mathrm{B}_{k+t-1}(p)$ and the faces of $\mathrm{Z}_{k, l, t}(p)$ that are not in $\mathrm{B}_{k+t-1}(p)$. Furthermore, $f_{e} \mapsto f_{e}^{\prime}$ takes notch faces to notch faces and radial faces to radial faces; also, if $f_{1}$ and $f_{2}$ are two consecutive faces of $B$, then their common vertex along $\partial \mathrm{B}_{k+t-1}(p)$, call it $v$, has codegree one or two and this determines whether or not $f_{1}$ and $f_{2}$ share an edge incident to $v$. Therefore $B$ is obtained from $Z_{k, l, t}(p)$ by making zero or more identifications along $\partial Z_{k, l, t}(p)$. If there are no identifications, then we have that $B=\mathrm{Z}_{k, l, t}(p)$. We also get that $B=B_{k+t}(p)$ because no face outside of $B$ can have a vertex of distance $k+t-1$ from $p$ and so we are done. So now, in Case 1 say that there are edges on $\partial Z_{k, l, t}(p)$ that are identified and in Case 2 say that no edges along $\partial Z_{k, l, t}(p)$ are identified but that there are vertices that are identified.

Case 1: Assume that $e_{1}$ and $e_{2}$ are on $\partial \mathrm{Z}_{k, l, t}(p)$ and are identified in going from $\mathrm{Z}_{k, l, t}(p)$ to $B$. If $e_{i}$ is not incident to a central ray of $Z_{k, l, t}(p)$, then $e_{i}$ has one endpoint that is on $B_{k+t-1}(p)=\mathrm{Z}_{k, l, t-1}(p)$, is not a curvature vertex, and has degree 4 in $\mathrm{Z}_{k, l, t}(p)$. Any identification with another vertex of the same color would yield a vertex of degree more than four, a contradiction. Thus $e_{1}$ and $e_{2}$ are both incident to central rays of $\mathrm{Z}_{k, l, t}(p)$. Because the rotational symmetry has only two fixed points (i.e., the poles), the $n$ endpoints of the central rays of $\mathrm{Z}_{k, l, t}(p)$ must either correspond to $n$ distinct vertices in $B$ or one vertex in $B$ that is fixed under the $n$-fold rotational symmetry (that is, the other pole of the rotational symmetry, call it $q$ ). We assume the latter is true as this is the only way in which edges of $\partial Z_{k, l, t}(p)$ may be identified. Now the $2 n$ edges of $\partial Z_{k, l, t}(p)$ incident to the central rays are identified as per the numbering in Figure 23 to obtain $Z^{\prime}$.

There are two subcases to consider here: in Case 1.1 say that $2 \leq l \leq k-1$ and in Case 1.2 say that $l \in\{1, k\}$.

Case 1.1: Now $\partial Z^{\prime}$ consists of $n$ vertex-disjoint cycles. Since $q$ is black, the black vertices on $\partial Z^{\prime}$ all have degree 4 in $Z^{\prime}$ and the white vertices on $\partial Z^{\prime}$ all have degree 2 in $Z^{\prime}$ except 


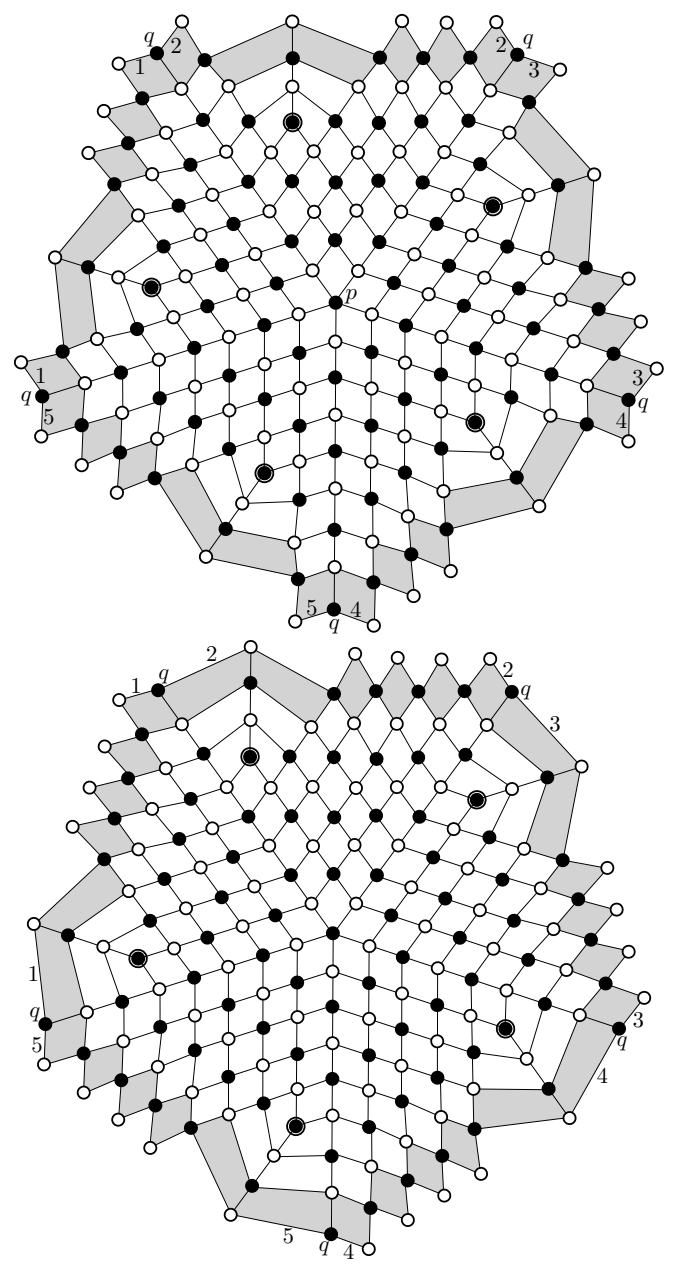

Figure 23: The disk $Z^{\prime}$ for $l=2$ and $l=1$, respectively.

for exactly $2 n$ white vertices on $\partial Z^{\prime}$ having degree 3 in $Z^{\prime}$ : the $n$ endpoints of the curvature rays along with the $n$ neighbors of $q$. As shown at the beginning of Case 1, no two edges of $\partial Z^{\prime}$ may be identified and so $B$ is obtained from $Z^{\prime}$ by the identification of zero or more pairs of white vertices having degree 2 in $Z^{\prime}$ to obtain white vertices of degree 4 in $G$. We cannot, of course, identify white vertices from two distinct cycles of $\partial Z^{\prime}$ because this would create a non-separating cycle in the embedding of $G$ in the sphere, a contradiction.

Consider a cycle $C$ in $\partial Z^{\prime}$ with vertices $w_{1}, b_{1}, w_{2}, b_{2}, \ldots, w_{m}, b_{m}$ and say by way of contradiction that $w_{i}$ and $w_{j}$ are identified in going from $Z^{\prime}$ to $B$. Call the resulting faceconnected subsurface after this identification $Z^{\prime \prime}$. Now $w_{i}, b_{i}, b_{j-1}$ (and also $w_{i}, b_{i-1}, b_{j}$ ) all have degree four in $Z^{\prime \prime}$. Because $2 \leq l \leq k-1$, we now get that these three vertices are on a common face of $G$ and so $w_{i+1}$ and $w_{j-1}$ (and also $w_{i-1}$ and $w_{j+1}$ ) must be identified in going from $Z^{\prime \prime}$ to $B$. This identification process will continue and eventually yield a contradiction by either: trying to identify a white vertex of degree 3 with a white 
vertex of degree 2 or 3 , by creating a face of length four having a white vertex of positive codegree on its boundary, or by creating a cycle of length 2 .

Thus we must have that $Z^{\prime}=B$; however, this is not possible for the following reason. Consider a cycle $C$ in $\partial B$ and let $P$ be the disk of $G$ with $\partial P=C$. The black vertices on $C$ have degree 2 in $P$, the white vertices on $C$ have degree 4 in $P$ save for two of them which have degree 3 in $P$. By the rotational symmetry, the interior vertices of $P$ include exactly one black degree- 3 vertex and all other interior vertices have degree 4 in $P$. Thus $P$ has an odd number of vertices of odd degree, a contradiction.

Case 1.2: As in Case 1.1, $\partial Z^{\prime}$ consists of $n$ vertex-disjoint cycles and the black vertices on $\partial Z^{\prime}$ all have degree 4 in $Z^{\prime}$. However, in this case, the white vertices on $\partial Z^{\prime}$ all have degree 2 in $Z^{\prime}$ except for the $n$ white vertices adjacent to $q$ which have degree 4 in $Z^{\prime}$. Let $C$ be one cycle in $\partial Z^{\prime}$. As in Case 1.1, no two edge of $C$ may be identified in going from $Z^{\prime}$ to $B$. Label the vertices in rotational order along $C$ with $w_{1}, b_{1}, \ldots, w_{m}, b_{m}$ where $w_{1}$ is the white vertex of $C$ having degree 4 in $Z^{\prime}$. Since $b_{m}, w_{1}, b_{1}$ all have degree 4 , they must be on the same face of $G$ and so we must have that $w_{m}=w_{2}$. This in turn implies that $w_{m-1}=w_{3}$, etc. These identifications are not possible, however, because $m=2 k-2$ and $k$ is even and thus these identifications would create a face of length 2 , a contradiction.

Case 2: In this case, the only possible identifications along $\partial Z_{k, l, t}(p)$ in going from $\mathrm{Z}_{k, l, t}(p)$ to $B$ are pairs of vertices which have degree 2 in $\mathrm{Z}_{k, l, t}(p)$. Say that the vertices on $\partial \mathrm{Z}_{k, l, t}(p)$ of distance $k+t+1$ from $p$ have color $\kappa$ and the vertices of distance $k+t$ have color $\lambda$. Hence $\{\kappa, \lambda\}=\{$ black, white $\}$ (e.g., in Figure $21 \kappa=$ white). The vertices of color $\kappa$ on $\partial \mathrm{Z}_{k, l, t}(p)$ have degree 2 in $\mathrm{Z}_{k, l, t}(p)$ save for the $n$ endpoints of the curvature rays (which have degree 3 in $Z_{k, l, t}(p)$ ) and the vertices of color $\lambda$ on $\partial Z_{k, l, t}(p)$ have degree 4 in $Z_{k, l, t}(p)$ save for the $n$ endpoints of the central rays (which have degree 3 in $\left.\mathrm{Z}_{k, l, t}(p)\right)$.

Label the vertices along $\partial Z_{k, l, t}(p)$ in rotational order with $\lambda_{1}, \kappa_{1}, \ldots, \lambda_{m}, \kappa_{m}$ in which $\lambda_{1}$ is the endpoint of a central ray. Say that $\kappa_{i}$ and $\kappa_{j}$ are identified in $B$, and say that $v_{1}, \ldots, v_{n}$ is the orbit of $\kappa_{i}$ under the rotational symmetry and $u_{1}, \ldots, u_{n}$ the orbit of $\kappa_{j}$. Then $\left|\left\{u_{1}, \ldots, u_{n}, v_{1}, \ldots, v_{n}\right\}\right|=2 n$ in $Z_{k, l, t}(p)$ and $\left|\left\{u_{1}, \ldots, u_{n}, v_{1}, \ldots, v_{n}\right\}\right|=n$ or 1 in $G$. It cannot be that $\left|\left\{u_{1}, \ldots, u_{n}, v_{1}, \ldots, v_{n}\right\}\right|=1$ in $G$ because then these $2 n$ degree-2 vertices in $\mathrm{Z}_{k, l, t}(p)$ would then identify to one vertex of degree $4 n$ in $G$, a contradiction. Thus $\left|\left\{u_{1}, \ldots, u_{n}, v_{1}, \ldots, v_{n}\right\}\right|=n$ in $G$. Because of the rotational symmetry these two orbits of vertices must alternate along the cycle $\partial Z_{k, l, t}(p)$ and because $G$ is spherical, identified pairs of vertices (e.g., $\kappa_{i}$ and $\kappa_{j}$ ) must appear consecutively along $\partial Z_{k, l, t}(p)$.

Let $\gamma_{i j}$ be the $\kappa_{i} \kappa_{j}$-path along $\partial Z_{k, l, t}(p)$ which contains no other vertices from $v_{1}, \ldots, v_{n}, u_{1}, \ldots, u_{n}$. Again, because of the rotational symmetry, at most one endpoint of a curvature ray and at most one endpoint of a central ray occurs on $\gamma_{i j}$. Suppose that $\lambda_{i+1}$ and $\lambda_{j}$ are the neighbors of $\kappa_{i}=\kappa_{j}$ on $\gamma_{i j}$. If $\lambda_{i+1}$ and $\lambda_{j}$ both have degree 4 in $\mathrm{Z}_{k, l, t}(p)$, then $\kappa_{i}, \lambda_{i+1}$, and $\lambda_{j}$ must all be on the same face of $G$ and so we must have that $\kappa_{i+1}=\kappa_{j-1}$ in $B$. Similarly if $\lambda_{i}$ and $\lambda_{j+1}$ both have degree 4 in $Z_{k, l, t}(p)$, then $\kappa_{i-1}=\kappa_{j+1}$ in $B$. These identifications of degree- $2, \kappa$-colored vertices must continue in each direction along $\partial Z_{k, l, t}(p)$ until either we reach the endpoint of a curvature ray or central ray. Thus $\gamma_{i j}$ contains either the endpoint of a curvature ray or the endpoint of a central ray, but not both.

We claim that $\gamma_{i j}$ contains the endpoint of a curvature ray and not the endpoint of a central ray. This is because if the latter were true, then, because the endpoint of a central 
ray is of color $\lambda$, this identification of $\kappa$-colored vertices along $\gamma_{i j}$ would end with either a face of length two (a contradiction) or a face of length four containing a $\kappa$-colored vertex of degree 2 (again a contradiction).

Now since the endpoint of a curvature ray is contained in $\gamma_{i j}$, the identification of $\kappa$ colored vertices along $\gamma_{i j}$ ends with the identification of some $\kappa_{a-1}$ and $\kappa_{a+1}$ where $\kappa_{a}$ is the endpoint of the curvature ray and so has degree 3 in $G$. Also note that this implies that $\kappa_{a}$ is the midpoint of $\gamma_{i j}$. Identifications of $\kappa$-colored vertices from $\kappa_{i}=\kappa_{j}$ that are off of $\gamma_{i j}$ must then stop at the endpoint of the central rays. These identifications of vertices in $\mathbf{Z}_{k, l, t}(p)$ result in the disk $\widehat{Z}_{k, l, t}(p)$. These are the only vertex identifications that can happen in going from $Z_{k, l, t}(p)$ to $B$ because we started with an arbitrary vertex identification. Thus $B=\widehat{Z}_{k, l, t}(p)$ and we must also have that $B=\widehat{Z}_{k, l, t}(p)=\mathrm{B}_{k+t}(p)$ because any face of $G$ outside of $\widehat{Z}_{k, l, t}(p)$ cannot contain a vertex of distance $k+t-1$ from $p$.

Proposition 4.8 gives us the three possible structures for $B_{k+t}(p)$. The proof of Proposition 4.8 is similar to the proof of Proposition 4.2 using Proposition 4.7 in the place of Proposition 4.1 .

Proposition 4.8. Let $k \geq 1$ be odd and let $O_{1}$ be one orbit of $n$ degree-3 curvature vertices contained in $\partial \mathrm{B}_{k}(p)$. Let $\mathrm{O}_{2}$ be the second orbit of $n$ degree-3 curvature vertices of $G$. Let $t \geq 0$ be such that $B_{k+t-1}(p)$ contains no curvature vertices aside from $O_{1} \cup\{p\}$ and $\mathrm{B}_{k+t}(p)$ contains $\mathrm{O}_{2}$. One of the following holds.

(1) If $t$ is odd (that is, $k+t$ is even), then $\mathrm{B}_{k+t}(p)=\mathrm{Z}_{k, l, t}(p)$ and the vertices of $\mathrm{O}_{2}$ are the endpoints of the central rays of $\mathrm{B}_{k+t}$.

(2) If $t$ is even (that is, $k+t$ is odd), then either

(a) $\mathrm{B}_{k+t}(p)=\widehat{\mathrm{Z}}_{k, l, t}(p)$ and the vertices of $\mathrm{O}_{2}$ are the endpoints of the curvature rays and appear in the interior of $\mathrm{B}_{k+t}(p)$, or

(b) $\mathrm{B}_{k+t}(p)=\mathrm{Z}_{k, l, t}(p)$ and the vertices of $\mathrm{O}_{2}$ are on $\partial \mathrm{B}_{k+t}(p)$ but not the endpoints of the central rays or curvature rays.

Proposition 4.9. If $\mathrm{B}_{k+t}(p)=\mathrm{Z}_{k, l, t}(p)$ is as given in Proposition 4.8(2)(b), then $G$ is given by the Four-Parameter Construction with uniquely determined parameters.

Proof. Consider two consecutive central rays of $Z_{k, l, t}(p)$ along with the vertices, edges and faces of $\mathrm{Z}_{k, l, t}(p)$ between these central rays, that is, the closure of a fundamental region of the rotational symmetry, call it $F$. A rendering of $F$ is shown on the left in Figure 24 in which the dashed lines have length $t$ (with $t=0$ a possibility) and are identified.

Let $o_{1} \in O_{1}$ and $o_{2} \in O_{2}$ be the vertices of $O_{1} \cup O_{2}$ in $F$. Since $o_{2}$ is not on the endpoint of the central ray or curvature ray, $o_{2}$ appears on $\partial Z_{k, l, t}(p)$ in one of the two circled areas shown on the left of the figure; take the right of Figure 24 as an illustration. We may assume without loss of generality that $o_{2}$ is in the upper region because if $o_{2}$ is in the lower circled region on the left of Figure 24, then we may reflect $Z_{k, l, t}(p)$ to get $Z_{k, k-l+1, t}(p)$ and then have $\mathrm{O}_{2}$ in the upper region.

Now take the fundamental region $F^{\prime}$ adjacent to and in the counterclockwise direction from $F$. The rendering of $F \cup F^{\prime}$ shown in Figure 25 is geometrically flat and so we coordinatize in the obvious way with $p$ at $(0,0)$. As a result, the grey lines shown in Figure 25 

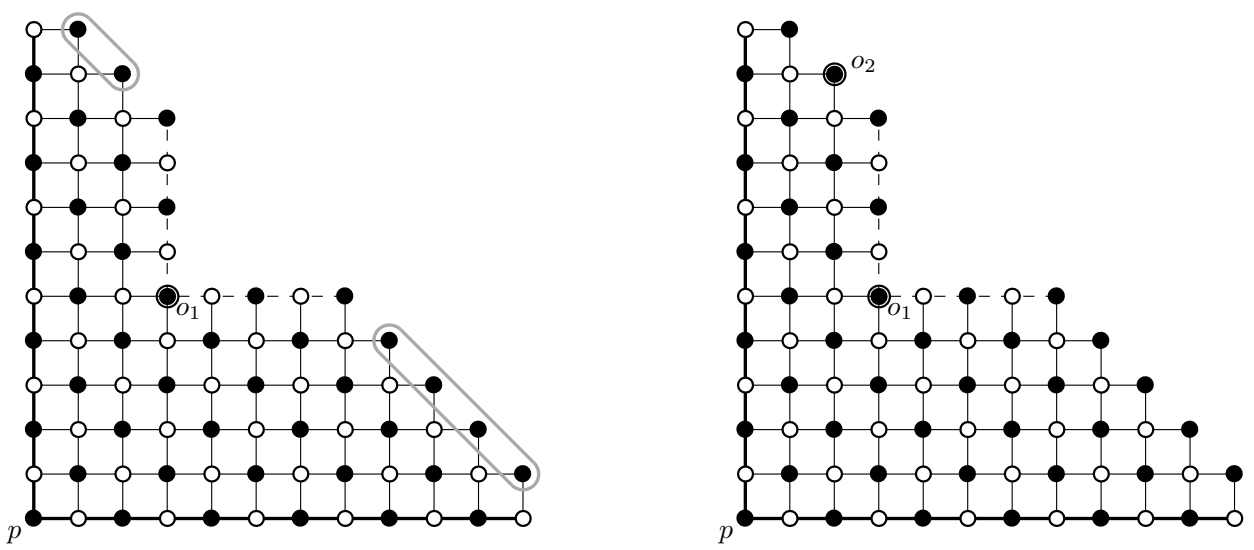

Figure 24: The fundamental region $F$.

form a quadrilateral with a right angle at the origin. Evidently the quadrilateral is also convex because each of the interior angles is less than $180^{\circ}$. Hence the grey quadrilateral is of the type used in the Four-Parameter Construction; furthermore, the parameters defining this quadrilateral are uniquely determined by the positions of $o_{1}$ and $o_{2}$ within $F$. Hence $\mathrm{Z}_{k, l, t}(p)$ contains $n$ of these special quadrilaterals at $p$.

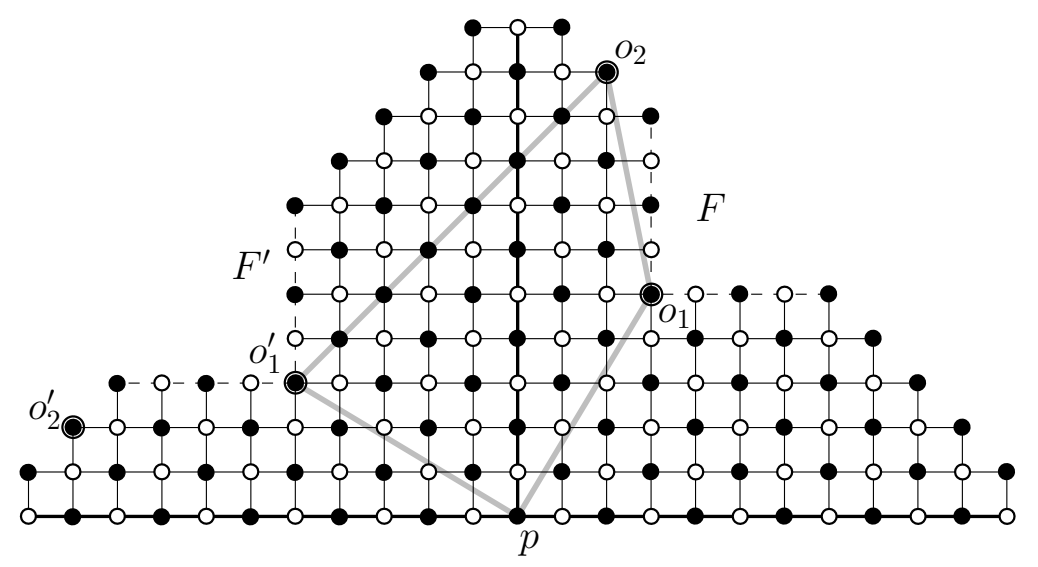

Figure 25: Two fundamental regions $F$ and $F^{\prime}$ along with a four-parameter special integer quadrilateral.

Conversely, by Proposition 4.7, the entire Four-Parameter Construction using this quadrilateral must contain $\mathrm{Z}_{k, l, t}(p)$ because the positions of the curvature vertices in the Four-Parameter Construction come from the corners of the quadrilaterals. So one possibility for $G$ is given by the Four-parameter construction. Now, we will show that there is only one spherical quadrangulation which contains $\mathrm{Z}_{k, l, t}(p)$ and has $\mathrm{O}_{2}$ in this position on $\partial \mathrm{Z}_{k, l, t}(p)$, which will complete our proof.

Consider the vertices on the boundary $\partial Z_{k, l, t}(p)$. The black vertices on this boundary 
have degree 2 in $Z_{k, l, t}(p)$ save for the $n$ endpoints of the curvature rays which have degree 3 . The white vertices on this boundary have degree 4 in $Z_{k, l, t}(p)$ save for the $n$ endpoints of the central rays which have degree 3 . Let $w_{1}, b_{1}, c_{1}, \ldots, w_{n}, b_{n}, c_{n}$ be vertices in clockwise rotational order on $\partial \mathrm{Z}_{k, l, t}(p)$ in which the $w_{i}$ 's are the endpoints of the central rays, $b_{i}$ 's the vertices of $\mathrm{O}_{2}$, and $c_{i}$ 's the endpoints of the curvature rays.

Now let $D$ be the disk of $G$ constructed from the faces of $G$ not in $\mathrm{Z}_{k, l, t}(p)$. Thus $D$ contains the pole $q \neq p$ and $\partial D=\partial \mathrm{Z}_{k, l, t}(p)$; furthermore, by rotational symmetry the pole $q$ is in the interior of $D$. Now,

- the $w_{i}$ 's, $b_{i}$ 's, and $c_{i}$ 's all have degree 3 in $D$,

- the remaining black vertices on $\partial D$ have degree 4 in $D$, and

- the remaining white vertices on $\partial D$ have degree 2 in $D$.

By Proposition 2.8, we may consider $D$ as a subgraph of a standard $r$-disk $S_{r}$ with a black central vertex, call it $q_{0}$, for some large enough value of $r$. Of course the embedding of $D$ must have $q$ corresponding to $q_{0}$ and, since $q$ is in the interior of $D$, the central rays of $D$ must lie on the central rays of $S_{r}$. Thus the embedding of $D$ in $S_{r}$ is unique up to dihedral symmetry. For the uniqueness of $D$ as a completion of $G$, we need to show that there is no other disk $D^{\prime}$ in $S_{r}$ having $n$-fold rotational symmetry around $q$ with a bijection between the vertices of $\partial D^{\prime}$ and $\partial D$ which respects degrees and cyclic ordering.

Consider the black vertices of $S_{r}$ and connect pairs of black vertices on the same face with an edge (say it is also black). This black graph is a quadrangulation with every internal vertex of degree 4 aside from $q$ which has degree $n$. Call any transverse path in the black graph a black diagonal path of $S_{r}$ or $D$. Call the $n$ black diagonal paths of $S_{r}$ or $D$ that originate from $q$ the diagonal rays of $S_{r}$ or $D$.

Now consider the boundary faces of $D$ and the black diagonal edges in each. These black edges form a cycle, call it $C$, in the black-diagonal graph and $C$ is contained entirely inside the disk $D$. Note that the cycle $C$ consists of black diagonal paths whose endpoints are the $c_{i}$ 's, $b_{i}$ 's, and $w_{i}^{\prime}$ 's where $w_{i}^{\prime}$ is the black neighbor of $w_{i}$ that is not in $\mathrm{Z}_{k, l, t}(p)$. Traversing $C$ in $S_{r}$ with $q$ to our right, the $c_{i}$ 's and $b_{i}$ 's represent a right turn rather than a transverse path and the $w_{i}^{\prime}$ 's represent a left turn. We will now show that $C$ (and hence the boundary faces of $D$ ) is uniquely determined by the positions of $c_{i}$ 's, $b_{i}$ 's, and $w_{i}^{\prime}$ 's on $\partial D$. This will imply the uniqueness of $D$.

Now let $V$ be the region of $S_{r}$ between and including two consecutive diagonal rays, call them $Y_{1}$ and $Y_{2}$ in the clockwise direction. The intersection of $C$ with $Y_{1}$ has one or more connected components, each of which is either an isolated vertex or a path of positive length. If there is no path of positive length, then let $y_{1}$ be some vertex of $C$ on $Y_{1}$. If there is a path of positive length in the intersection, then let $y_{1}$ be the last vertex of some intersection path when traversing $C$ in the clockwise direction. Let $y_{2}$ be the corresponding vertex on $Y_{2}$ under the rotational symmetry in the clockwise direction, and let $P$ be the $y_{1} y_{2}$-path in $C$ in the clockwise direction. Consider the square $Q$ in $V$ given by the black diagonals of $S_{r}$ shown in Figure 26.

In the clockwise traversal of $C, P$ contains two right turns and one left turn and at the rest of the vertices of $P$, a transverse crossing. The sequence of turns is either left-right-right, right-left-right, or right-right-left; however, if necessary we can reflect $R$ around the axis $Y_{1}$ and reverse the traversal of $C$ so that the first turn is right. In Figure 26, $V$ is rendered as part of the standard $4 \times 4$ grid in the $x y$-plane between the perpendicular lines $y=x$ 


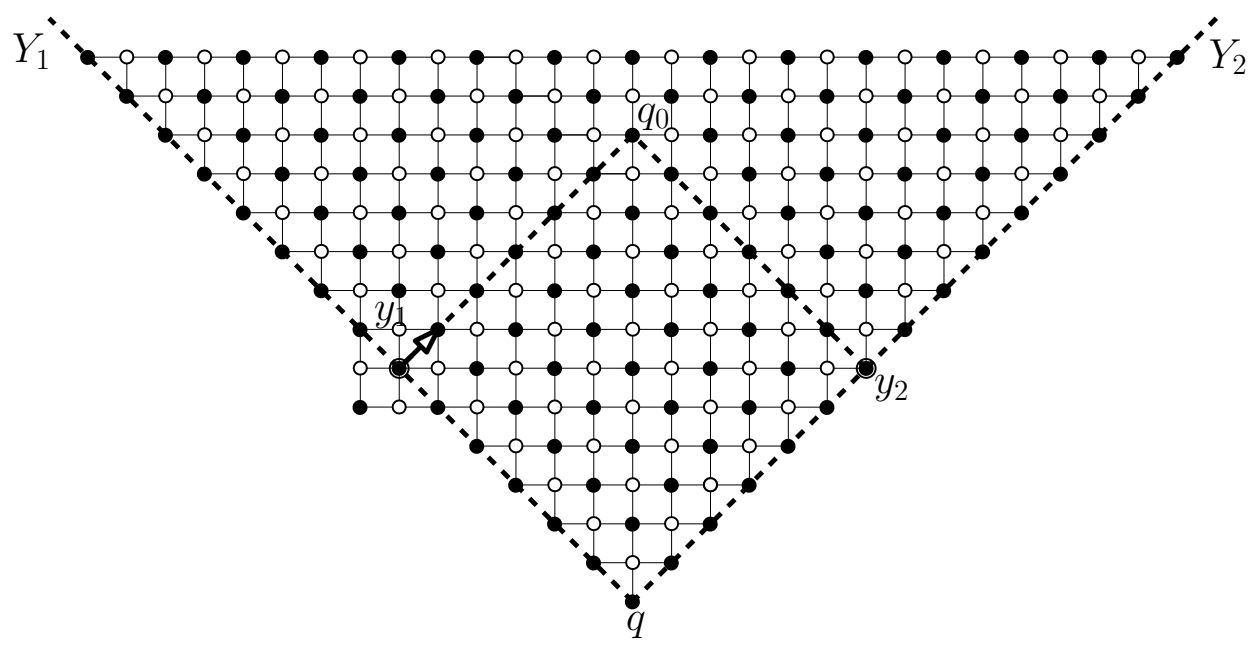

Figure 26: The square defined by $Y_{1}, y_{1}, Y_{2}$, and $y_{2}$ in $V$.

and $y=-x$. In the traversal of $P$, two right turns and a left turn yield a net change of $90^{\circ}$ degrees in the clockwise direction. Because of the way in which $y_{1}$ is chosen, the first edge of $P$ is in the direction of the arrow shown in Figure 26. Now the path $P$ is in $V$ and is completely determined by the placement of the two right turns in the 3 -turn sequence. In Figure 27 we have three examples of $P$. Since these turns are determined by the placements of the $c_{i}$ 's, $b_{i}$ 's, and $w_{i}$ 's on $\partial D$, there is only one possibility for $P$ and so for $C$ and hence for $D$.

Proposition 4.10. If $\mathrm{B}_{k+t}(p)=\widehat{\mathrm{Z}}_{k, l, t}(p)$ is as given in Proposition 4.8(2)(a), then $G$ is given by the Four-Parameter Construction with uniquely determined parameters.

Proof. As in the proof of Proposition 4.9, consider two adjacent fundamental regions $F$ and $F^{\prime}$ of $\widehat{Z}_{k, l, t}(p)$ between three consecutive central rays. These may be rendered in a geometrically flat fashion as in Figure 28 with identically labeled vertices being identified in $G$ and appropriate identifications of dashed edges. Note that the path of dashed edges has positive length. Since the rendering is flat we have a special integer quadrilateral as used in the Four-Parameter Construction with parameters uniquely determined by $k, l$, and $t$ as shown in the figure. Therefore the Four-Parameter Construction yields one possibility for $G$. In order to show that this is the only possibility for $G$, we will show that there is only one possibility for the disk in $G$ around $q$ sharing its boundary with $\widehat{Z}_{k, l, t}(p)$.

Consider the dashed edge shown in Figure 29 along with its orbit of $n$ edges under the rotational symmetry. Let $\widetilde{Z}$ be the disk around $p$ consisting of $\widehat{Z}_{k, l, t}(p)$ along with the $n$ faces bounded by these $n$ edges and $\widehat{Z}_{k, l, t}(p)$.

Let $D$ be the disk defined by the faces of $G$ not contained in $\widetilde{Z}$. Note that $D$ contains $q$ in its interior and $\partial D=\partial \widetilde{Z}$. All of the white vertices of $\partial D=\partial \widetilde{Z}$ have degree 4 in $\widetilde{Z}$ and degree 2 in $D$. Among the black vertices of $\partial D=\partial \widetilde{Z}, n$ have degree 3 in both $\widetilde{Z}$ and $D$ and the rest have degree 2 in $\widetilde{Z}$ and degree 4 in $D$.

Say that $l$ is the smallest distance in $G$ from the pole $q$ to any vertex on $\partial D=\partial \widetilde{Z}$. Let $u$ be one such vertex on the common boundary. It must be that $\mathrm{d}(u, p)=k+t+1$ rather 

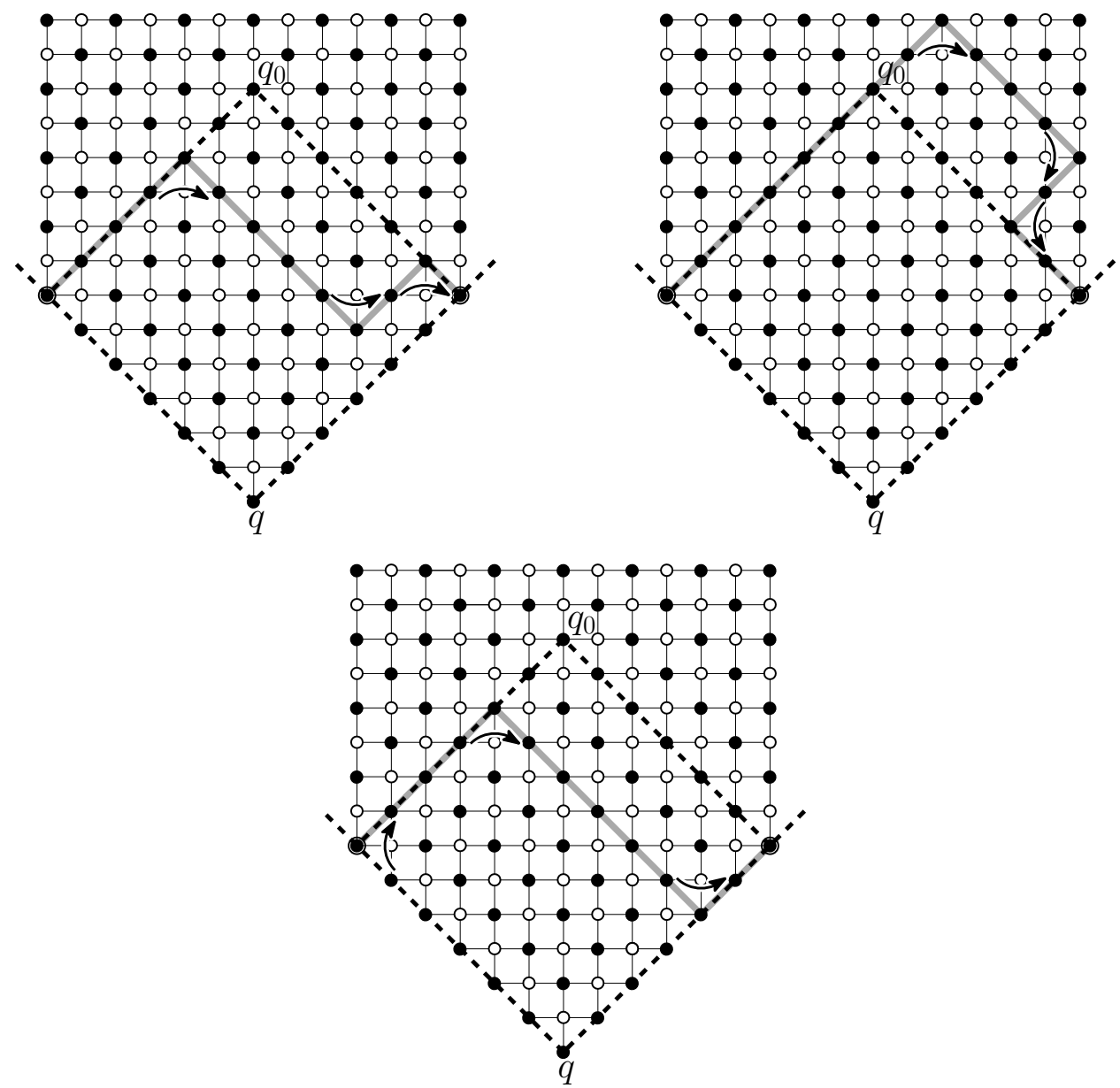

Figure 27: Three examples of the path $P$. In the third example, $B$ intersects $Y_{1}$ in a path.

than $k+t$. This is because the vertices of $\partial B_{k+t}(p)$ of distance $k+t$ from $p$ are all white and are saturated by $\widetilde{Z}$ and so any path from $q$ to one of these vertices of distance $k+t$ from $p$ must go through the vertices of distance $k+t+1$ from $p$. Therefore $u$ is black and has degree 2 or 3 in $\widetilde{Z}$.

Since $u$ is black, $l$ must be even. First suppose that $u$ can be chosen to be in $\mathrm{B}_{l-1}(q)$ (which by Proposition 4.1 is a standard $(l-1)$-disk); that is, $u$ is a vertex of degree 2 on the boundary of $\mathrm{B}_{l-1}(q)$. Since the white vertices of $\partial \mathrm{B}_{l-1}(q)$ have distance $l-1$ from $q$ and $l$ is the smallest distance of a vertex from $q$ to $\widetilde{Z}$, these white vertices on $\partial \mathrm{B}_{l-1}(q)$ are not in $\widetilde{Z}$. So any black vertex on $\partial \mathrm{B}_{l-1}(q)$ which is identified to a black vertex on $\partial \widetilde{Z}$ forces another identification of two black boundary vertices. Eventually these identifications will run to the degree- 3 vertices of $\widetilde{Z}$ on $\partial \widetilde{Z}$. But this forces these black vertices to have degree 5 in $G$, a contradiction. 


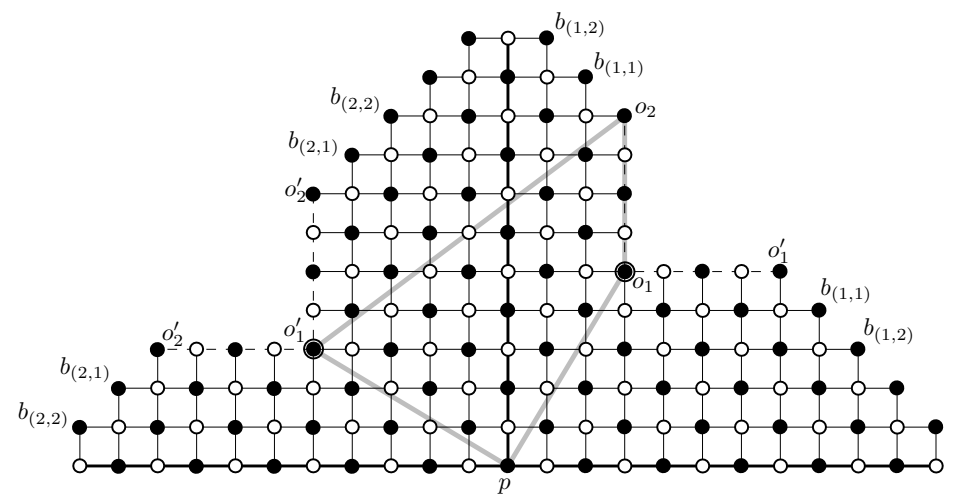

Figure 28: A flat rendering of two fundamental regions for $\widehat{Z}_{k, l, t}(p)$.

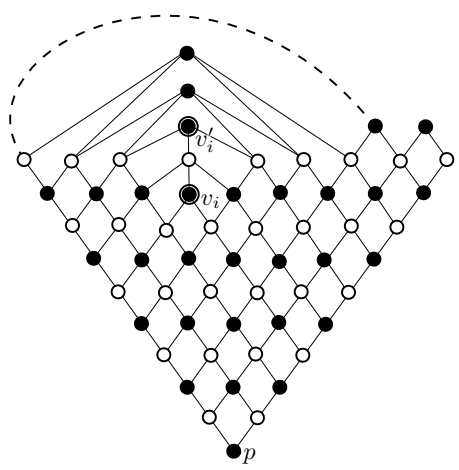

Figure 29: There are $n$ edges in $G$ with endpoints in $\widehat{Z}_{k, l, t}(p)$. One such edge is shown as a dashed curve.

Thus $u$ is a vertex of degree 3 on the boundary of $\mathrm{B}_{l}(q)$; that is, $u$ is the endpoint of a central ray of $\mathrm{B}_{l}(q)$ which by Proposition 4.1 is a standard $l$-disk. Degree considerations now force $u$ and the vertices in its orbit to be identified with the degree- 3 vertices of $\widetilde{Z}$ on $\partial \widetilde{Z}$. From here $\partial \mathrm{B}_{l}(q)$ must then be identified with $\partial \widetilde{Z}$.

Proposition 4.11. If $\mathrm{B}_{k+t}(p)=\mathrm{Z}_{k, l, t}(p)$ is as given in Proposition 4.8(1), then $G$ is given by the Four-Parameter Construction with uniquely determined parameters.

Proof. Again, as in the proof of Proposition 4.9, consider two adjacent fundamental regions $F$ and $F^{\prime}$ of $Z_{k, l, t}(p)$ between three consecutive central rays rendered in a geometrically flat fashion as in Figure 30. Again we have a special integer quadrilateral contained in $F \cup F^{\prime}$ as shown in the figure with uniquely determined parameters. Therefore the FourParameter Construction yields one possibility for $G$, and we will now show that there is only one possibility for the disk in $G$ around $q$ sharing its boundary with $\mathrm{Z}_{k, l, t}(p)$.

Let $m$ be the shortest distance from $q$ to a vertex $u$ on $\partial Z_{k, l, t}(p)$. Since all of the black vertices on $\partial Z_{k, l, t}(p)$ are saturated by $Z_{k, l, t}(p)$, it must be that $u$ is white and hence $m$ is odd. By Proposition 4.1 and the definition of $m, \mathrm{~B}_{m}(q)$ is a standard $m$-disk. If $u$ is not the endpoint of a central ray of $\mathrm{B}_{m}(q)$, then $u$ is saturated by $\mathrm{B}_{m}(q)$. Since $u$ has degree 


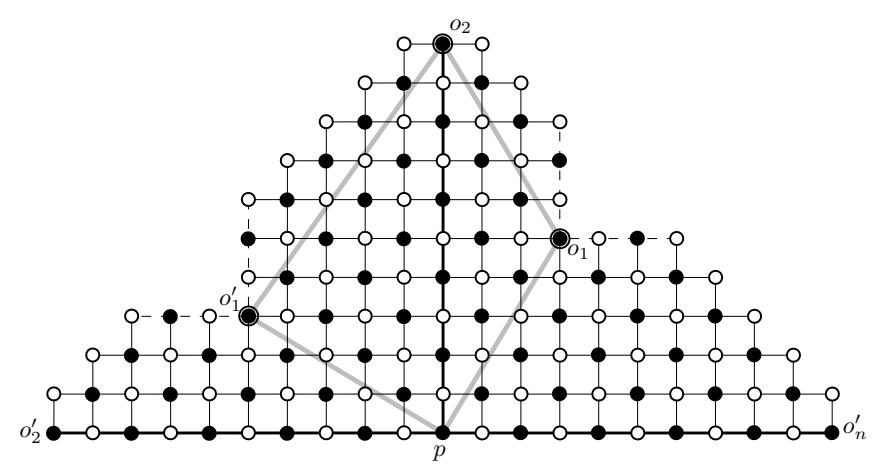

Figure 30: A flat rendering of two fundamental regions for $\mathrm{Z}_{k, l, t}(p)$.

4 in $G$, it must be that $u$ has degree 2 in $\partial \mathrm{Z}_{k, l, t}(p)$ and that the boundary edges incident to $u$ in $\mathrm{B}_{m}(q)$ are identified to the boundary edges incident to $u$ in $\partial \mathrm{Z}_{k, l, t}(p)$. The only boundary edges of both disks that are left are those incident to the central rays of $\mathrm{B}_{m}(q)$ and the curvature rays of $\partial Z_{k, l, t}(p)$. Degree considerations and the fact that all faces must have length 4 now force all edges of $\partial \mathrm{B}_{m}(q)$ to be identified to all edges of $\partial \mathrm{Z}_{k, l, t}(p)$.

\section{ORCID iDs}

Lowell Abrams (iD https://orcid.org/0000-0002-8174-5957

Daniel Slilaty (D) https://orcid.org/0000-0002-7918-3641

\section{References}

[1] L. Abrams and D. Slilaty, Cellular automorphisms and self-duality, Trans. Am. Math. Soc. 367 (2015), 7695-7773, doi:10.1090/tran/6258.

[2] D. Archdeacon and S. Negami, The construction of self-dual projective polyhedra, J. Comb. Theory Ser. B 59 (1993), 122-131, doi:10.1006/jctb.1993.1059.

[3] D. Archdeacon and R. B. Richter, The construction and classification of self-dual spherical polyhedra, J. Comb. Theory Ser. B 54 (1992), 37-63, doi:10.1016/0095-8956(92)90065-6.

[4] M. Deza and M. Dutour Sikirić, Geometry of Chemical Graphs: Polycycles and Two-Faced Maps, volume 119 of Encyclopedia of mathematics and its Applications, Cambridge University Press, Cambridge, 2008, doi:10.1017/cbo9780511721311.

[5] J. E. Graver and E. J. Hartung, Self-dual spherical grids, Electron. J. Comb. 21 (2014), Paper 1.36, 36, doi:10.37236/3510.

[6] A. Márquez, A. de Mier, M. Noy and M. P. Revuelta, Locally grid graphs: classification and Tutte uniqueness, Discrete Math. 266 (2003), 327-352, doi:10.1016/s0012-365x(02)00818-x.

[7] B. Servatius and H. Servatius, Self-dual maps on the sphere, volume 134, pp. 139-150, 1994, doi:10.1016/0012-365x(93)e0069-g.

[8] B. Servatius and H. Servatius, The 24 symmetry pairings of self-dual maps on the sphere, Discrete Math. 140 (1995), 167-183, doi:10.1016/0012-365x(94)00293-r.

[9] B. Servatius and H. Servatius, Self-dual graphs, Discrete Math. 149 (1996), 223-232, doi: $10.1016 / 0012-365 \times(94) 00351-i$. 
[10] C. Thomassen, Tilings of the torus and the Klein bottle and vertex-transitive graphs on a fixed surface, Trans. Amer. Math. Soc. 323 (1991), 605-635, doi:10.2307/2001547. 